\title{
Thermodynamic fluctuations in solar photospheric three-dimensional convection simulations and observations ${ }^{\star}$
}

\author{
C. Beck ${ }^{1,2,3}$, D. Fabbian ${ }^{1,2}$, F. Moreno-Insertis ${ }^{1,2}$, K. G. Puschmann ${ }^{4}$, and R. Rezaei ${ }^{5}$ \\ 1 Instituto de Astrofísica de Canarias (IAC), Calle Vía Láctea s/n, 38205 La Laguna, Tenerife, Spain \\ 2 Departamento de Astrofísica, Universidad de La Laguna (ULL), 38206 La Laguna, Tenerife, Spain \\ 3 National Solar Observatory (NSO), 3010 Coronal Loop, 88349 Sunspot, New Mexico, USA \\ e-mail: [cbeck; damian; fmi]@iac.es \\ ${ }^{4}$ Leibniz-Institut für Astrophysik Potsdam (AIP), An der Sternwarte 16, 14482 Potsdam, Germany \\ e-mail: kgp@aip.de \\ 5 Kiepenheuer-Institut für Sonnenphysik (KIS), Schöneckstr. 6, 79104 Freiburg, Germany \\ e-mail: rrezaei@kis.uni-freiburg.de
}

Received 28 March 2013 / Accepted 23 June 2013

\section{ABSTRACT}

\begin{abstract}
Context. Numerical three-dimensional (3D) radiative (magneto-)hydrodynamical [(M)HD] simulations of solar convection are nowadays used to understand the physical properties of the solar photosphere and convective envelope, and, in particular, to determine the Sun's photospheric chemical abundances. To validate this approach, it is important to check that no excessive thermodynamic fluctuations arise as a consequence of the partially incomplete treatment of radiative transfer causing radiative damping that is too modest.

Aims. We investigate the realism of the thermodynamics in recent state-of-the-art 3D convection simulations of the solar atmosphere carried out with the Stagger code.

Methods. We compared the characteristic properties of several Fe I lines $(557.6 \mathrm{~nm}, 630 \mathrm{~nm}, 1565 \mathrm{~nm})$ and one Si I line at $1082.7 \mathrm{~nm}$ in solar disc-centre observations of different spatial resolution with spectra synthesized from 3D convection simulations. The observations were taken with ground-based (Echelle spectrograph, Göttingen Fabry-Pérot Interferometer (GFPI), POlarimetric LIttrow Spectrograph, Tenerife Infrared Polarimeter, all at the Vacuum Tower Telescope on Tenerife) and space-based instruments (Hinode/Spectropolarimeter). We degraded the synthetic spectra to the spatial resolution of the observations, based on the distribution of the continuum intensity $I_{\mathrm{c}}$. We estimated the spectral degradation to be applied to the simulation results by comparing atlas spectra with averaged observed spectra. In addition to deriving a set of line parameters directly from the intensity profiles, we used the SIR (Stokes Inversion based on Response functions) code to invert the spectra.

Results. The spatial degradation kernels yield a similar generic spatial stray-light contamination of about $30 \%$ for all instruments. The spectral stray light inside the different spectrometers is found to be between $2 \%$ and $20 \%$. Most of the line parameters from the observational data are matched by the degraded HD simulation spectra. The inversions predict a macroturbulent velocity $v_{\text {mac }}$ below $10 \mathrm{~m} \mathrm{~s}^{-1}$ for the HD simulation spectra at full spatial resolution, whereas they yield $v_{\text {mac }} \lesssim 1000 \mathrm{~m} \mathrm{~s}^{-1}$ at a spatial resolution of 0 '.3. The temperature fluctuations in the inversion of the degraded HD simulation spectra do not exceed those from the observational data (of the order of $100-200 \mathrm{~K} \mathrm{rms}$ for $-2 \lesssim \log \tau_{500 \mathrm{~nm}} \lesssim-0.5$ ). The comparison of line parameters in spatially averaged profiles with the averaged values of line parameters in spatially resolved profiles indicates a significant change in (average) line properties on a

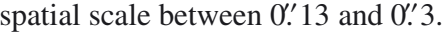

Conclusions. Up to a spatial resolution of $0 ! 3$ (GFPI spectra), we find no indications of excessive thermodynamic fluctuations in the 3D HD simulation. To definitely confirm that simulations without spatial degradation contain fully realistic thermodynamic fluctuations requires observations at even higher spatial resolution (i.e. $<0$ '! 13).
\end{abstract}

Key words. Sun: photosphere - methods: data analysis - line: profiles

\section{Introduction}

The abundances of chemical elements in the solar atmosphere are crucial as a reference for chemical abundances throughout the universe, with a profound influence on planetary science, stellar evolution theory, or the interpretation of stellar spectra. Solar abundances are commonly derived from comparisons between observed spectral lines and theoretical spectra from atmospheric models with a varying degree of sophistication (see Asplund et al. 2005, and references therein). Such models range from static one-dimensional (1D) atmospheres treated in local

\footnotetext{
$\star$ Appendices A and B are available in electronic form at http://www. aanda.org
}

thermal equilibrium (LTE) to dynamical three-dimensional (3D) atmospheres with a non-LTE treatment (e.g. Shchukina et al. 2012).

For static 1D solar atmosphere models, such as those of Holweger \& Mueller (1974), Vernazza et al. (1981), Fontenla et al. (2006), and variations thereof, one must first adopt values for the solar abundance of the various chemical elements to derive a temperature stratification through the comparison of observed and synthetic spectra, using both the locations of bound-free edges, i.e. the limiting wavelength of relevant ionization transitions, and the shape of spectral lines. However, the retrieved temperature stratifications of the atmospheres are then used to derive the abundances by comparison of up to a few tens 
of resulting synthetic spectral lines to observations. This is therefore partly a case of circular reasoning, with the abundances as both input and output parameters.

Another problem of static 1D models is that the dynamical nature of the solar atmosphere cannot be consistently taken into account. Resolved or unresolved velocities can only be partly included in the static 1D models by using generic macroturbulent $\left(v_{\mathrm{mac}}\right)$ and microturbulent $\left(v_{\mathrm{mic}}\right)$ velocities. These are free parameters that show a certain degree of degeneracy when one tries to disentangle different contributions to the formation process of spectral lines (Allende Prieto et al. 2001).

The accuracy of the information about dynamical variations provided by observations is also always limited, since observed spectra suffer from the combined effects of limited spatial, spectral, and temporal resolution. The accurate retrieval of all thermodynamic parameters needed for an exact characterization of the dynamics, such as temperature, mass velocities, and mass densities, is therefore only partly possible on the observational side.

To obtain a realistic dynamical model of the solar atmosphere, one thus has to resort to numerical simulations. The growing available computing power has made it possible to run increasingly large-scale and higher-sampling multi-dimensional calculations solving the complex set of coupled differential equations necessary to model the relevant physics in the solar photosphere (e.g. Nordlund \& Stein 1990; Steiner et al. 1998; Stein \& Nordlund 1998). When one attempts, however, to extend such simulations to cover also the solar chromosphere, nonLTE effects appear that cannot be (fully) included in 3D numerical simulations that are of a sufficiently large scale while remaining computationally feasible (cf. Rammacher \& Cuntz 1991; Skartlien 2000; Asplund et al. 2003; Leenaarts et al. 2007, 2009; Hayek et al. 2010; Wedemeyer-Böhm \& Carlsson 2011; Carlsson \& Leenaarts 2012; Leenaarts et al. 2012). Similarly, a possible problem of photospheric simulations is that radiative transfer through the atmosphere can only be included in an approximate way, commonly by using opacity binning (e.g. Nordlund 1982; Skartlien 2000; Vögler 2004; Vögler et al. 2004).

Nonetheless, the results of such numerical simulations are considered realistic enough to be used in approaches to determine solar abundances (e.g. Asplund et al. 2000). The advantages of these simulations are that they cover the dynamics of mass motions without the need to resort to ad-hoc parameters such as $v_{\text {mac }}$ or $v_{\text {mic }}$. Furthermore, they directly contain all information, including especially the gas density, on an absolute geometrical height scale, while, e.g., spectral line inversions just provide information of physical parameters on an optical depth scale. The conversion to a geometrical height scale is in the latter case not straightforward, needing sophisticated methods and certain assumptions commonly in the form of a boundary condition on electron (or gas) pressure or density (e.g. Sánchez Almeida 2005; Puschmann et al. 2005, 2010; Beck 2011).

However, the use of 3D hydrodynamical (HD) simulations coupled with improved atomic data and differences in equivalent width measurements retrieved from observations resulted in the course of the last decade in a downward revision of the solar abundance of mainly C, N and O (Asplund et al. 2005, 2009), which significantly deteriorated the previous agreement between predicted and observed sound speeds in the solar interior derived from helioseismology (Bahcall et al. 2005). We note that, where available, non-LTE corrections (e.g. Fabbian et al. 2009a,b) were included in the abundance determination from simulations (cf. Asplund et al. 2009, and references therein).
More recently, magnetic fields have been suggested as another source of appreciable uncertainty (Fabbian et al. 2010, 2012).

Kalkofen (2012) made an interesting comment about the use of simulation results as a reference. The atomic abundances are usually derived by matching the observed solar spectrum with the space- and/or time-averaged synthetic line profiles from the simulations. In the averaging process, the thermodynamic properties of the simulations are smeared out. Because of the simplified treatment of the radiative transfer in the simulations, an important damping factor could be missing. This could lead to unrealistic/excessive thermodynamic fluctuations both in temperature and mass motions. As a consequence of, e.g., too large velocity fluctuations, the average predicted profile would become too broad, thus leading to a downward revision of the solar abundance reference of a given chemical element, as derived by matching observational spectra. The argument by Kalkofen (2012) is based on the fact that the intrinsic thermodynamic parameters in simulations at full spatial resolution were investigated only partially up to now.

The agreement of synthetic and observed data was verified in terms of the contrast of continuum intensity, both at disc centre and for its centre-to-limb (CLV) variation (e.g. Asplund et al. 2000; Wedemeyer-Böhm \& Rouppe van der Voort 2009; Asplund et al. 2009; Afram et al. 2011), as well as in terms of the match between average synthetic and observed profiles (e.g. Asplund et al. 2000). Spatially resolved profiles were later also studied for comparison purposes (Pereira et al. 2009c). All of these comparisons, however, have a clear limitation: the observations employed always have a lower spatial resolution than the original simulations, whose spatial grid size can be as small as several $\mathrm{km}$. The good match with observations that was achieved in the aforementioned studies therefore proves that the simulations can be spatially and spectrally degraded to the properties of the observations, but it actually does not exclude excessively large thermodynamic fluctuations in the simulations themselves, as pointed out by Danilovic et al. (2008).

In this paper, we investigate the realism of the thermodynamics in numerical 3D convection simulations by comparing disc-centre observations in several spectral lines with the results from simulations. This shall serve as the first step in a thorough derivation of the solar oxygen abundance using the same set of simulations. In a subsequent study, we will turn to the CLV of selected spectral lines and in the continuum, which can allow one to calibrate the efficiency of $\mathrm{H}$ collisions in non-LTE calculations or to constrain the temperature stratification.

Sections 2 and 3, respectively, describe the observations and the modelling employed here. The data reduction and the analysis methods are explained in Sect. 4. Results are presented in Sect. 5 and are discussed in Sect. 6. Section 7 provides our conclusions. The Appendices contain the results for some of the spectral lines not discussed in the main text.

\section{Observations}

We used several quiet-Sun (QS) observations at disc centre taken with different instruments and telescopes (see Table 1). We introduce them here divided into three groups:

(a) The infrared range. For all spectral lines in the (near)infrared (IR) range (Si I $1082.7 \mathrm{~nm}$, Fe I $1564.8 \mathrm{~nm}$, Fe I $1565.2 \mathrm{~nm}$ ), the Tenerife Infrared Polarimeter (TIP; Martínez Pillet et al. 1999; Collados et al. 2007) at the German Vacuum Tower Telescope (VTT; Schröter et al. 1985) was employed. The data in these lines were largearea scans. The data at $1083 \mathrm{~nm}$ were taken during a velocity 
Beck et al.: Thermodynamic fluctuations in photospheric convection simulations and observations

Table 1. Instruments and data properties, and corresponding settings of the spectral synthesis for the simulations.

\begin{tabular}{l|cccccc|c}
\hline \hline Instrument & SP & GFPI & POLIS & TIP & TIP & Echelle & Simulation \\
\hline$\lambda$ [nm] & 630 & 630 & 630 & 1083 & 1565 & 557 & all \\
Main line(s) & Fe I & Fe I & Fe I, O I & Si I & Fe I & Fe I, O I & Fe I, Si I \\
Dispersion [pm] & 2.15 & 2.31 & 1.49 & 2.10 & 2.95 & 0.31 & 0.50 \\
Sampling ["] & $0.3 \times 0.3$ & $0.11 \times 0.11$ & $0.5 \times 0.15$ & $0.5 \times 0.18$ & $0.5 \times 0.18$ & $0.18 \times 0.18$ & $0.13 \times 0.13$ \\
FOV ["] & $115 \times 115$ & $30 \times 53$ & $100 \times 94$ & $100 \times 78$ & $40 \times 75$ & $35 \times 200$ & $8.3 \times 8.3$ \\
Integration time [s] & 1.6 & 0.64 & 3.3 & 3.3 & 4 & 3 & - \\
Spatial resolution" ["] & $0.64^{2}$ & $0.3 / 0.6^{3}$ & 0.7 & 0.7 & 0.7 & $\sim 1$ & $0.13^{4}$ \\
App. \# of profiles & 150.000 & 140.000 & 130.000 & 90.000 & 30.000 & 220.000 & 4.000 \\
\hline
\end{tabular}

Notes. ${ }^{(1)}$ Estimated values, cf. Sect. 4.3 below; (2) sampling-limited resolution; ${ }^{(3)}$ different data reduction methods, estimated spatial resolution values from PuB11; ${ }^{(4)}$ sampling $\equiv$ resolution.

calibration campaign of the CHROmospheric TELescope (CHROTEL; Bethge et al. 2011) on 08 December 2007 between UT 10:02:35 and 10:17:55. The data at $1565 \mathrm{~nm}$ were taken on 21 May 2008 from UT 08:44:18 until 08:51:43, in between the two long-integrated data sets used in Beck \& Rezaei (2009), and cover the same field of view (FOV) as the data presented in the latter article. Spatial and spectral sampling, total FOV, the integration time and the approximate number of profiles in the FOV are listed in Table 1. The integration time denotes the total exposure time that entered in a single measurement of the full Stokes vector at one wavelength position or one slit position.

(b) The $630 \mathrm{~nm}$ lines. The data in the Fe I line at $630.25 \mathrm{~nm}$, taken with the Göttingen Fabry-Pérot Interferometer (GFPI; Puschmann et al. 2006; Bello González \& Kneer 2008) at the VTT on 07 June 2009, are described in detail in Puschmann \& Beck (2011, PuB11). We use the same spectral scan as those authors, with data reduced on the one hand with the multi-object multi-frame blind deconvolution (MOMFBD; van Noort et al. 2005) method, and on the other hand only destretched to a reference image that was speckle-reconstructed with the method of Puschmann $\&$ Sailer (2006). The spectral line was sampled on 28 wavelength steps. The data at $630 \mathrm{~nm}$ from the POlarimetric LIttrow Spectrograph (POLIS; Beck et al. 2005a,b) were taken simultaneously to the TIP data in $1082.7 \mathrm{~nm}$ on 08 Dec. 2007. Bethge et al. (2012) discuss another example of data taken with this setup on the same day. The Hinode/Spectropolarimeter (SP; Kosugi et al. 2007; Tsuneta et al. 2008) map was taken during HOP 0190 on 05 Sep. 2011 between UT 07:34:50 and 07:59:03. The FOV was in this case located slightly off disc centre at a heliocentric angle of less than 10 degrees, which should, however, not have a strong impact on the spectra.

(c) The $557 \mathrm{~nm}$ range. All the observations described so far provided Stokes vector measurements that allow one to locate any magnetic flux of sufficient strength inside the FOV and to determine the magnetic field properties. Data from a wavelength range around $557 \mathrm{~nm}$ were used in addition. They correspond to a part of the CLV observations that we collected as an ingredient to be used in the derivation of the solar oxygen abundance (Fabbian et al., in prep.), as a reappraisal of previous literature estimates (cf. Asplund et al. 2004; Meléndez \& Asplund 2008; Pereira et al. 2009c,a). These spectra were obtained with the Echelle spectrograph of the VTT at high spectral resolution $(\lambda / \Delta \lambda \sim 1000000)$, but without polarimetry. Additionally to an OI line at $557.7 \mathrm{~nm}$, the spectral range encompassed also the strong Fe I line at $557.6 \mathrm{~nm}$. We therefore included in the present study a disc-centre data set that we collected on 21 Nov. 09 between UT 08:50 and 09:05.

All ground-based observations profited from the real-time correction of seeing by the Kiepenheuer-Institut adaptive optics (AO) system (von der Lühe et al. 2003) at the VTT.

\section{Convection simulations and spectral synthesis}

The synthetic spectra used in our analysis are based on a set of 3D solar convection simulations obtained and described by Fabbian et al. $(2010,2012)$ that were carried out using the Stagger code (cf. Stein et al. 2011; Kritsuk et al. 2011; Beeck et al. 2012). The simulation box spanned approximately $6 \times$ $6 \mathrm{Mm}^{2}$ horizontally, covering $\sim 15$ granules at any given time, with 252 equidistant points in each direction, resulting in a horizontal grid spacing of $\sim 24 \mathrm{~km}$. The height range was covered by 126 grid points, reaching from $\sim 0.5 \mathrm{Mm}$ above the height corresponding to the optical depth unity $\left(\tau_{500 \mathrm{~nm}}=1\right)$ level down to $\sim 2.0 \mathrm{Mm}$ below it, with non-uniform vertical sampling having a finest spacing of about $15 \mathrm{~km}$.

The Stagger code uses a sixth-order finite differences spatial scheme and a third-order Runge-Kutta temporal scheme to solve the equations for the conservation of mass, momentum and energy. At the same time, the radiative transfer equation is solved assuming LTE, in this instance along nine rays (one per octant, plus the vertical). Four opacity bins were employed to model the wavelength dependency of the radiation field. For more details on the opacity binning technique adopted in the code see Nordlund (1982), Collet et al. (2011) and Beeck et al. (2012).

The effective temperature in the simulation snapshots is controlled by the (fixed) entropy density condition imposed on fluid flowing in from the bottom boundary. This parameter was adjusted so that the resulting $T_{\text {eff }}$ in the atmospheric models (about $5730 \mathrm{~K}$ ) was within less than one percent of the solar effective temperature derived from observations. Fabbian et al. (2012, their Fig. 2) have shown that the continuum intensity computed for the same series of snapshots as employed here agrees reasonably well with literature data, albeit being slightly $(\geqq 5 \%)$ lower (cf. also the bottom left panel in Fig. 8 below). The variation of the emerging radiative flux at the surface for the different (magneto)-hydrodynamical [(M)HD] cases corresponds to an uncertainty on $T_{\text {eff }}$ of the order of $0.2 \%$ only, i.e. about $10 \mathrm{~K}$. The simulation runs covered the field-free purely HD case and three MHD cases with a vertical unipolar magnetic flux density of 50,100 , and $200 \mathrm{G}$, respectively.

In the present investigation we selected only the last snapshot of the statistically stationary regime of each (M)HD run. The spectral synthesis was performed using the LTE code LILIA 
(Socas-Navarro 2001). For this step, the appropriate input data corresponding to the chosen snapshot were prepared by trimming the simulation results to a vertical extent ranging from $\sim+425 \mathrm{~km}$ above the average $\tau_{500 \mathrm{~nm}}=1$ level to $\sim-475 \mathrm{~km}$ below it, and by interpolating to a grid of equidistant points over the new physical depth. Those heights correspond to an optical depth range from about $\log \tau_{500 \mathrm{~nm}} \approx-4$ to $\log \tau_{500 \mathrm{~nm}} \approx+2$, but with some variation across the FOV. The final input data cube had 115 depth points with a constant, finer vertical grid spacing of $\sim 7.8 \mathrm{~km}$, which was better suited to our spectral synthesis purpose.

Finally, for the spectral synthesis we also reduced the horizontal sampling to only include $63 \times 63$ grid points (without binning or averaging of the corresponding physical values) distributed over the same area as that of the original snapshot. Tests done for Fabbian et al. (2012) indicated that this approach does not introduce any kind of bias in the resulting set of synthetic spectra, while still providing a sufficiently large statistical base of about 4000 independent spectra. The final spatial sampling then corresponded to 0 ' $^{\prime} 13$, which is more than twice better than the best spatial resolution in the observations (cf. Table 1).

The spectral sampling for all synthetic lines was set to $0.5 \mathrm{pm}$. The synthetic spectra were computed using the Van der Waals broadening formula in the approximation of Unsöld (1955). The default solar abundance adopted by LILIA was used, i.e. the one given in Grevesse (1984), according to which the logarithmic solar abundance of iron is $\log (\mathrm{Fe})=7.5 \mathrm{dex}$ for the Sun, on the usual scale of $10^{12}$ atoms of hydrogen, i.e. $\log (\mathrm{H})=12.0$ dex.

The spectral lines considered in the present study form in the low to mid photosphere (cf. Cabrera Solana et al. 2005). All of them can be assumed to behave close to LTE except for the Si I line at $1082.7 \mathrm{~nm}$, where the line core is sensitive to non-LTE effects and to a lesser amount sensitive to 3D effects (e.g. Wedemeyer 2001; Shchukina \& Trujillo Bueno 2001; Shi et al. 2008; Shchukina et al. 2012; see also the corresponding sections in Asplund 2005). The line core of the Si I line also forms in the uppermost layers of the simulation box (up to 0.5 Mm height; Bard \& Carlsson 2008; Felipe et al. 2010) where the results of both the simulation and the spectral synthesis become less reliable.

\section{Data reduction and analysis methods}

\subsection{Intensity normalization}

For all observed or synthetic spectral lines up to $1.1 \mu \mathrm{m}$, we used the FTS atlas spectrum (Kurucz et al. 1984; Neckel 1999) as a reference. For spectra at longer wavelengths, data from the BASS2000 ${ }^{1}$ spectral database (Delbouille et al. 1973) were used. To correct for spectral intensity gradients in the observations (compare the blue-dotted and black curves in Fig. 1), we matched the continuum intensity of average observed spectra to the corresponding section of the atlas spectra in wavelength ranges outside (strong) spectral lines. Mismatches outside the wavelength range relevant for the derivation of the line parameters were ignored (e.g. the region at $630.07 \mathrm{~nm}$ in Fig. 1). The continuum intensity was normalized as in the case of the reference atlas spectra. The intensity normalization of all synthetic spectra from the simulations was always done fully analogously to the observations, i.e. with the atlas spectra as reference.

\footnotetext{
1 http://bass2000.obspm.fr/solar_spect.php
}

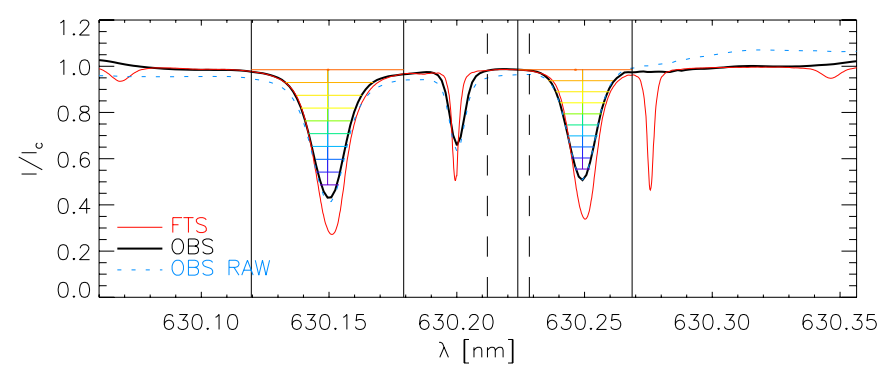

Fig. 1. Example spectrum at $630 \mathrm{~nm}$ from POLIS. Blue-dotted line: average spectrum without correction for intensity gradients over the wavelength range. Black line: corrected spectrum. Red line: FTS atlas spectrum. The dashed vertical lines denote the "continuum" range, the solid vertical lines the range that was considered for determining the line parameters of Fe I $630.15 \mathrm{~nm}$ and Fe I $630.25 \mathrm{~nm}$, respectively. The coloured horizontal lines inside the absorption profiles illustrate the method used to determine line properties at different line depression levels.

\subsection{Line parameters used}

To compare the thermodynamic properties of the observed and synthetic spectra, we used the following line parameters:

1. The line-core intensity $I_{\text {core }}$, i.e. the intensity at the deepest point of each absorption profile relative to the continuum intensity averaged over the full FOV.

2. The continuum intensity $I_{\mathrm{c}}$ in each profile.

3. The line depth, or, in other words, the difference $I_{\text {core }}-I_{\mathrm{c}}$ with both quantities evaluated for each spatially resolved profile.

4. The line-core velocity $v_{\text {core }}$, i.e. the velocity corresponding to the Doppler displacement of the location of minimal intensity relative to its average location in the FOV.

5. The relative line asymmetry, i.e. the relative difference of the areas enclosed between the line profile and the local continuum intensity level calculated on the blue or red side, respectively, of the line core. Calling those areas $A_{\mathrm{b}}$ and $A_{\mathrm{r}}$, the relative line asymmetry is then given by

$$
\delta A=\frac{A_{\mathrm{b}}-A_{\mathrm{r}}}{A_{\mathrm{b}}+A_{\mathrm{r}}} .
$$

6. The equivalent width, i.e. the total area of the absorption profile after its normalization to the average value of $I_{\mathrm{c}}$.

7. The full-width at half-maximum (FWHM) of the line, i.e. the FWHM of a Gaussian fit to the intensity profile.

8. A few line properties at ten equidistant line depression levels (as illustrated in Fig. 1) between the local continuum and the line core, namely, the line width, the bisector velocity, and the intensity value of each of the ten depression levels (called "bisector intensity" in the following). The intensity levels $I_{j}(x, y)(j=1 \ldots 10)$ were calculated by

$I_{j}(x, y)=I_{\text {core }}(x, y)+j / 10 \cdot\left(I_{\mathrm{c}}(x, y)-I_{\text {core }}(x, y)\right)$,

where $x$ and $y$ denote the pixel column and row inside the FOV, respectively. This calculation retrieves bisector properties at a constant line depth, which is assumed to correspond to layers of constant optical depth.

Figure 1 shows an example spectrum from the POLIS $630 \mathrm{~nm}$ data, with some of the relevant ranges and derived quantities. The continuum intensity was taken as the mean intensity inside the wavelength range limited by the two dashed lines. For the ground-based $630 \mathrm{~nm}$ data (POLIS, GFPI), we removed the telluric $\mathrm{O}_{2}$ blend at $630.27 \mathrm{~nm}$ by fitting a Gaussian to it that was 

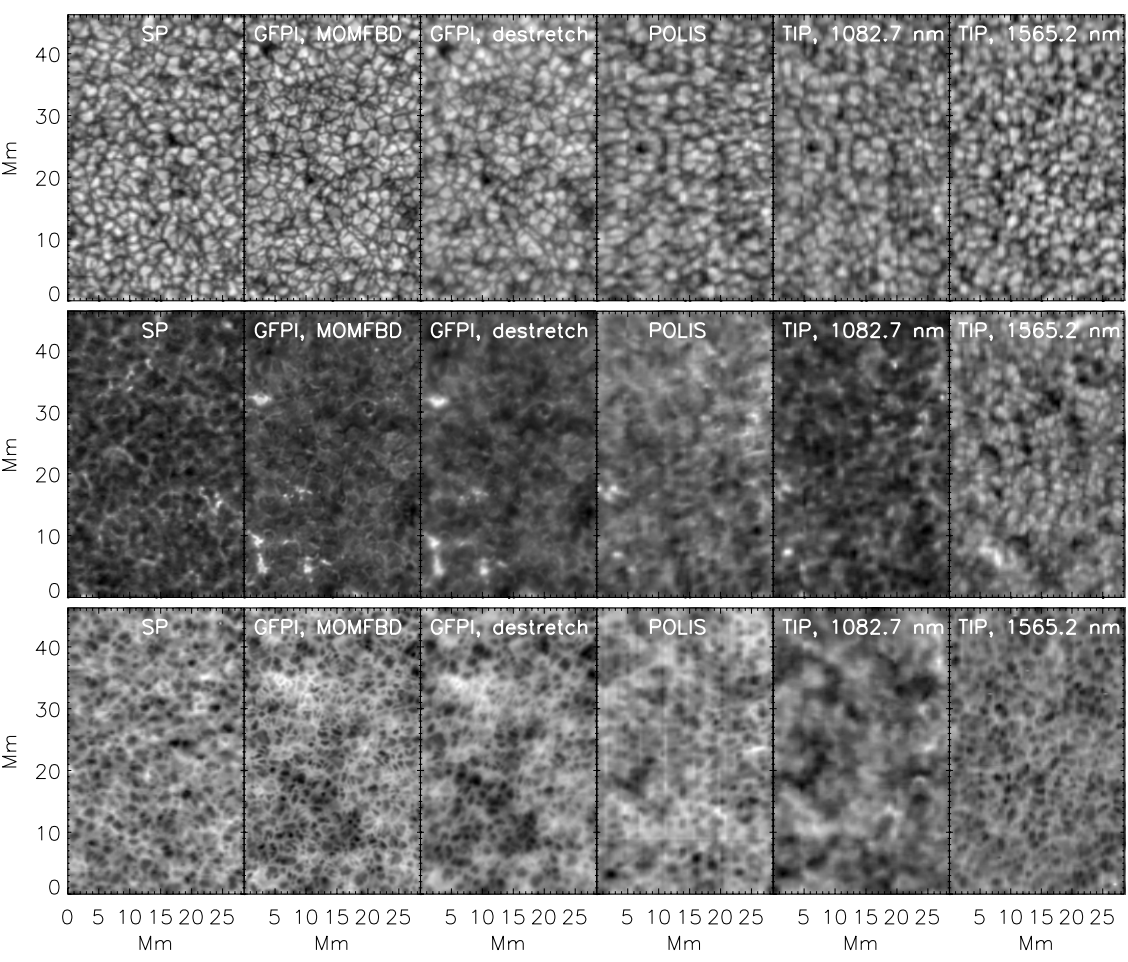

Fig. 2. Overview maps representative of the different observational data sets. Top to bottom: continuum intensity $I_{\mathrm{c}}$, line-core intensity $I_{\text {core }}$ and line-core velocity $v_{\text {core }}$. Left to right: SP, GFPI (MOMFBD), GPFI (destretched), POLIS, TIP@1083 nm, and TIP@1565 nm. Black/white in the velocity maps denotes motions towards/away from the observer. All images are scaled individually inside their full dynamical range. subsequently subtracted. We applied the same routine to observational and synthetic spectra to compute the corresponding line parameters, with the wavelength ranges adapted for use with each of the target lines in the corresponding wavelength regime $(557 \mathrm{~nm}, 630 \mathrm{~nm}, 1083 \mathrm{~nm}, 1565 \mathrm{~nm})$.

Figure 2 shows maps of three of the line parameters $\left(I_{\mathrm{c}}, I_{\text {core }}\right.$, $\left.v_{\text {core }}\right)$ for the six observational data sets obtained with the SP, GFPI, POLIS, and TIP. To facilitate a direct comparison in the figure, we extracted an equal-sized area of about $30^{\prime \prime}$ by $46^{\prime \prime}$ from each observation. The two different approaches to reduce the GPFI data (MOMFBD and destretching, second and third column) have an identical FOV albeit at different spatial resolutions. The shown sections of the POLIS data at $630 \mathrm{~nm}$ and of the TIP data at $1082.7 \mathrm{~nm}$ (fourth and fifth column) have been roughly aligned with one another to pixel precision to again facilitate an easier visual comparison, i.e. by obtaining a common FOV. All other data are neither co-spatial nor co-temporal. The maps show a clear variation in spatial resolution that approximately decreases from left to right, showing increasingly smeared structures. The MOMFBD GFPI data have the highest spatial resolution, the Echelle data at $557 \mathrm{~nm}$ (not shown here, see Fig. 7 below) have a significantly lower spatial resolution because of the seeing conditions during this observation.

\subsection{Spatial degradation}

To match the spatial resolution of simulations and observations, we determined suitable convolution kernels to degrade the simulations to the level of the observations (cf. Danilovic et al. 2008; Wedemeyer-Böhm \& Rouppe van der Voort 2009; Pereira et al. 2009c; Hirzberger et al. 2010; Afram et al. 2011). We used a kernel with two components, i.e. a Gaussian $K_{\mathrm{Gauss}}(r, \sigma)$ that is assumed to mainly reflect the spatial resolution and a Lorentzian $K_{\text {Lorentz }}(r, a)$ that should mimic the stray-light contribution by means of its extended wings (e.g. Mattig 1983), where $r$ denotes the radial distance from a given pixel. $\sigma$ is the variance of the Gaussian, and $a$ the parameter that regulates the shape of the Lorentzian. The final convolution kernel $K_{\text {total }}(r, \sigma, a)$ is then given by the convolution of the two kernels:

$$
\begin{aligned}
K_{\text {Gauss }}(r, \sigma) & =\frac{1}{\sqrt{2 \pi} \sigma} \exp ^{-\frac{r^{2}}{2 \sigma^{2}}} \\
K_{\text {Lorentz }}(r, a) & =\frac{1}{\pi} \frac{a}{r^{2}+a^{2}} \\
K_{\text {total }}(r, \sigma, a) & =K_{\text {Gauss }}(r, \sigma) * K_{\text {Lorentz }}(r, a),
\end{aligned}
$$

where $*$ denotes the convolution product.

As discussed in, e.g., Pereira et al. (2009c), the parameters $(\sigma, a)$ of the two kernels cannot be chosen independently. To prove that and to obtain a (semi-)automatic method for the derivation of the best parameter values, we quantified the mismatch between observations and the convolved simulations in the following way. We calculated histograms of the continuum intensity in all pixels in the FOV of each observation and in the spatially degraded HD simulation snapshot. Subsequently, we fitted Gaussian functions to all of the histograms (cf. Fig. 6 below for an example) from either the observations $\left(G_{\text {obs }}\right)$ or the degraded HD simulation $\left(G_{\text {degr }}\right)$. The fitted Gaussian curves were always shifted to a fixed reference central value and normalized by their maximum. The least-square value $\chi^{2}$ to be minimized was then defined using two contributions, one related to the deviation between the two Gaussian fits, and the other related to the difference between root-mean-squared (rms) continuum intensity contrasts. For asymmetric distributions the rms value and the width of the fitted Gaussian can differ significantly because a Gaussian fit mainly reproduces the core of a distribution, whereas the estimator of the rms takes also (far) outliers into account. We used a tenfold increased weighting of the rms contrast to achieve a comparable magnitude for both contributions. The $\chi^{2}$ was therefore set to

$$
\chi^{2}=\int_{I}\left(G_{\text {degr. }}(\sigma, a)-G_{\text {obs. }}\right)^{2} \mathrm{~d} I+10\left|\mathrm{rms}_{\text {degr. }}-\mathrm{rms}_{\text {obs. }}\right| .
$$

The $\chi^{2}$ was calculated for values of the FWHM of the $K_{\text {Gauss }}$ kernel (Eq. (2)) between 0.'12 and 1.5" and for $a$ in Eq. (3) 
Table 2. Top row: "best" degradation kernel parameters $a$ and $\operatorname{FWHM}(\sigma)$ for each instrument. Second row: average spatial stray light level $\alpha$ corresponding to each of those "best" kernels. Bottom rows: width of "best" kernel at three percentage levels of maximum amplitude.

\begin{tabular}{l|ccccccc}
\hline \hline & SP & GFPI, MOMFBD & GFPI, destr. & POLIS & TIP@ 1083 nm & TIP@ 1565 nm & ECHELLE@ 557 nm \\
\hline$a /$ FWHM & $0.16 / 0.59^{\prime \prime}$ & $0.68 / 0.12^{\prime \prime}$ & $1.56 / 0.19^{\prime \prime}$ & $1.32 / 0.34^{\prime \prime}$ & $0.28 / 0.96^{\prime \prime}$ & $0.92 / 0.59^{\prime \prime}$ & $1.92 / 1.33^{\prime \prime}$ \\
$\alpha$ & $28 \%$ & $19 \%$ & $30 \%$ & $31 \%$ & $34 \%$ & $33 \%$ & $40 \%$ \\
$50 \%$ & $0.39^{\prime \prime}$ & $0.34^{\prime \prime}$ & $0.37^{\prime \prime}$ & $0.39^{\prime \prime}$ & $0.42^{\prime \prime}$ & $0.39^{\prime \prime}$ & $0.45^{\prime \prime}$ \\
$25 \%$ & $0.58^{\prime \prime}$ & $0.47^{\prime \prime}$ & $0.55^{\prime \prime}$ & $0.58^{\prime \prime}$ & $0.74^{\prime \prime}$ & $0.66^{\prime \prime}$ & $0.87^{\prime \prime}$ \\
$10 \%$ & $0.81^{\prime \prime}$ & $0.56^{\prime \prime}$ & $0.81^{\prime \prime}$ & $0.87^{\prime \prime}$ & $1.16^{\prime \prime}$ & $1.00^{\prime \prime}$ & $1.55^{\prime \prime}$ \\
\hline
\end{tabular}

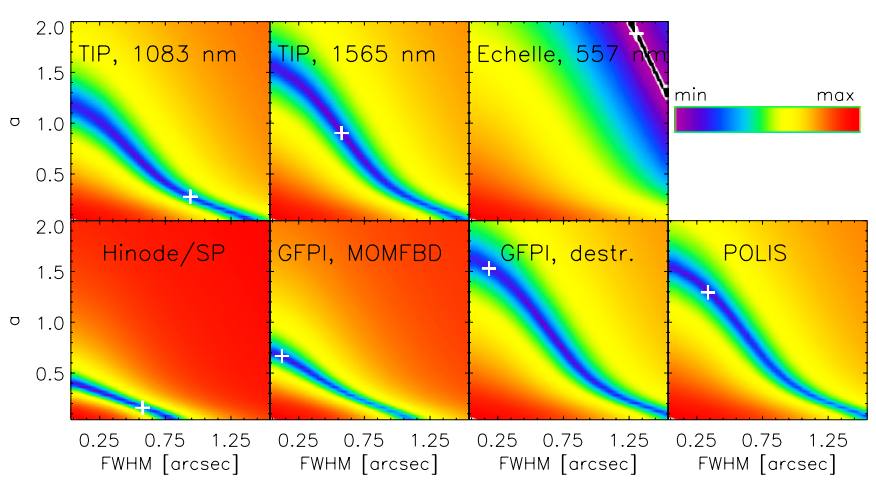

Fig. 3. $\chi^{2}$ values as a function of $\operatorname{FWHM}(=2 \sqrt{2 \log 2} \sigma)$ and $a$. Bottom row, left to right: SP, GFPI (MOMFBD), GFPI (destretched) and POLIS.Top row: TIP@1083 nm, TIP@1565nm and Echelle@557nm. The white crosses indicate the parameters corresponding to the "best" kernels.

between 0 and 2 , using for $G_{\text {obs. }}$ and $\mathrm{rms}_{\text {obs. }}$ the different observational data sets separately. The determination of the minimum of the resulting $\chi^{2}$-surface turned out to be a degenerate problem: as indicated by the blue/black coloured area in Fig. 3, for (nearly) each value of $\sigma$ a suitable value of $a$ can be found that yields a similarly small $\chi^{2}$. However, it is the parameter $\sigma$ that has the strongest influence on the resulting spatial resolution. We therefore selected "best" kernels by choosing the $\sigma$ value that provided the closest match in spatial resolution when visually comparing the observations and the degraded HD simulation (cf. Fig. 7 below). Having fixed $\sigma$, the minimum of the $\chi^{2}$ function provides the corresponding $a$. The white crosses in Fig. 3 denote the final choice of $(\sigma, a)$ for each observation (listed in the top row of Table 2).

Figure 4 shows the final "best" kernels for matching the convolved HD simulation with the various observations. The shape of the kernels fits the expectations when considering the visual impression of the data properties (Fig. 2). Namely, the convolution kernels for the ground-based spectrograph data and the destretched GFPI spectra have broader cores and higher wings than the SP and MOMFBD GFPI data, which leads to the lower contrast and lower spatial resolution of the former. The GPFI MOMFBD kernel has the smallest core of all, in agreement with its intensity map in Fig. 2 that shows more small-scale finestructure than any of our other observations.

The convolution kernels can be used to estimate the generic stray-light level in the observations. We calculated the total spurious intensity introduced into the central pixel of the simulation box from its surroundings (cf. Beck et al. 2011, BE11, their Eq. (8)) as

$I_{\text {stray }}(x, y, \lambda)=\sum_{x^{\prime} \neq x, y^{\prime} \neq y} K\left(x-x^{\prime}, y-y^{\prime}\right) I\left(x^{\prime}, y^{\prime}, \lambda\right)$,

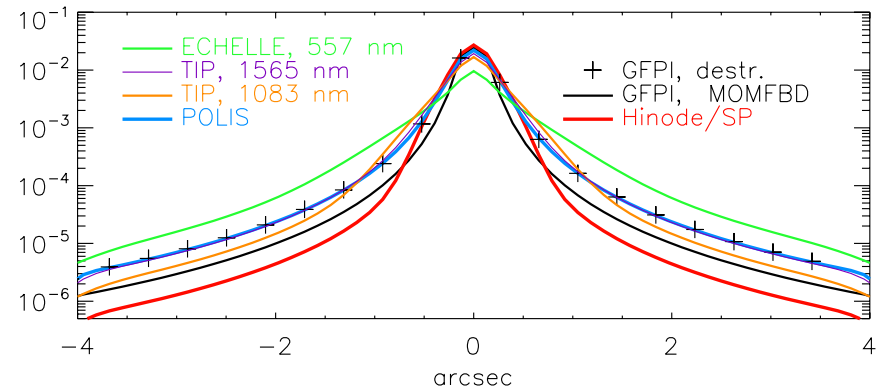

Fig. 4. "Best" degradation kernels $K_{\text {total }}$ for GFPI MOMFBD (black solid line), GFPI destretched (black crosses), Hinode/SP (thick red line), POLIS (thick blue line), TIP@ $1083 \mathrm{~nm}$ (orange line), TIP@ 1565 nm (purple line) and Echelle@557 nm (thick green line).

where $I\left(x^{\prime}, y^{\prime}, \lambda\right)$ are the synthetic spectra of the HD simulation at full resolution (HD-FR) and $x$ and $y$ denote the pixel column and row inside the FOV, respectively.

The generic spatial stray-light level $\alpha$ is then given by the value of $I_{\text {stray }}(x, y, \lambda)$ in a continuum wavelength range. The second row of Table 2 lists the average stray-light level for the various instruments expected for QS conditions. All spectrograph data (SP, POLIS, TIP, Echelle) and the destretched GFPI data yield a value $\gtrsim 30 \%$, with little to no dependence on the spatial resolution. The only clear reduction of stray light is seen for the deconvolved GFPI spectra, where in the deconvolution process some inverse kernel was already applied. Our generic straylight estimate encompasses both the effects of stray light from scattering on optics and from resolution effects caused by, e.g., the finite aperture size of the corresponding telescope (cf. for instance Danilovic et al. 2008). The contribution of resolution effects to stray light is present even for diffraction-limited observations and dominates the shape of the core of the kernel. For comparison, Wedemeyer-Böhm \& Rouppe van der Voort (2009) determined an effective value of $\alpha$ of $33 \%$ for the blue channel of the SOT/BFI onboard Hinode, of which about $8 \%$ should be caused by stray light and the rest by resolution effects (cf. Wedemeyer-Böhm 2008). For the SP, Danilovic et al. (2008) required a stray-light level of about $5 \%$ in addition to the theoretical point spread function (PSF) from the resolution effects to match the continuum contrast of their simulations and SP observations. Stray-light estimates for POLIS are listed in BE11, with a lower limit of $10 \%$ in umbrae of sunspots and about $24 \%$ in QS.

The width of the kernels at three percentage levels of the maximum kernel amplitude is listed in the lower rows of Table 2. The FWHM of the convolution kernel (row labelled 50\%) does not seem to be a good proxy for the spatial resolution because its variation across the different data sets is clearly too small, with a similar value of about 0.4 for all instruments. Only at the lower two levels $(25 \%, 10 \%)$ does the difference in quality become prominent, with observations of lower spatial resolution 


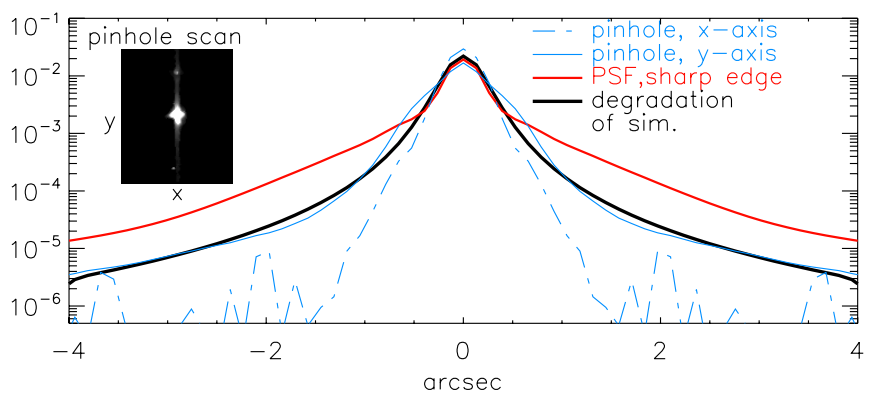

Fig. 5. Estimates of the PSF for POLIS from the degradation of the HD simulation (thick black line), a measurement of a knife-edge function (red line) and a scan across the pinhole in F1 (blue/blue-dot-dashed lines: cuts along $y / x$ ). The inset at the upper left shows the intensity map of the pinhole scan.

showing significantly broader kernels. For the ground-based slitspectrograph data (TIP, POLIS, Echelle; cf. the fifth row of Table 1 for the sampling-limited resolution), we accordingly used the width at $25 \%$ of the maximum kernel amplitude as an estimate of the spatial resolution.

The derivation of the degradation kernel by a convolution of simulations is an indirect method that implicitly assumes that the simulation can be used as reference for the true spatial intensity distribution. In the case of ground-based observations, the actual degradation has three contributions (e.g. Mattig 1971, 1983): the degradation by seeing fluctuations, by the telescope optics, and by the (instrument) optics behind the focal plane of the telescope (F1). The latter part, the instrumental PSF, can also be directly measured by some methods, usually using some type of (artificial) occulting disc in the telescope focal plane (cf. Briand et al. 2006; Wedemeyer-Böhm 2008; Beck et al. 2011; Löfdahl \& Scharmer 2012).

In the case of POLIS, we have an estimate of the PSF from an observation with a partly blocked FOV in F1 (BE11) and from a scan of a pinhole in F1 taken in April 2011. Figure 5 compares the convolution kernel derived for the HD simulation with the direct measurements of the PSF. The degradation of the HD simulation and the pinhole scan along the slit ( $y$-axis) yield nearly identical kernels. The small oscillation around the simulations' PSF likely represents the Airy pattern. The knife-edge measurement used in BE11 yields the same core, but has quite elevated wings compared to the other two methods. The cut in the scanning direction $(x)$ yields a smaller kernel that drops to zero faster than the one for the cut in $y$, because only light that is scattered already in front of the spectrograph is detected. The observation of a pinhole seems to be a practical approach for an observational determination of the instrumental PSF (cf. Löfdahl \& Scharmer 2012; Puschmann et al. 2012b), while the good match of the measured PSF with the one derived from degrading the simulation $^{2}$ suggests the latter as method of choice to obtain a PSF estimate otherwise.

Figure 6 shows the quality in the reproduction of the observed intensities by the degradation of the simulations with the respective "best" kernels. The intensity histograms of the data with high spatial resolution are asymmetric, with an extended tail towards intensities above unity (SP and GFPI MOMFBD data in the top row of Fig. 6). The deviation of the average value from unity seen in some cases is caused by the intensity

\footnotetext{
2 For the SP, the kernel derived from the degradation of the HD simulation matches a theoretical calculation of the PSF of the SOT (Asensio, priv. comm.).
}
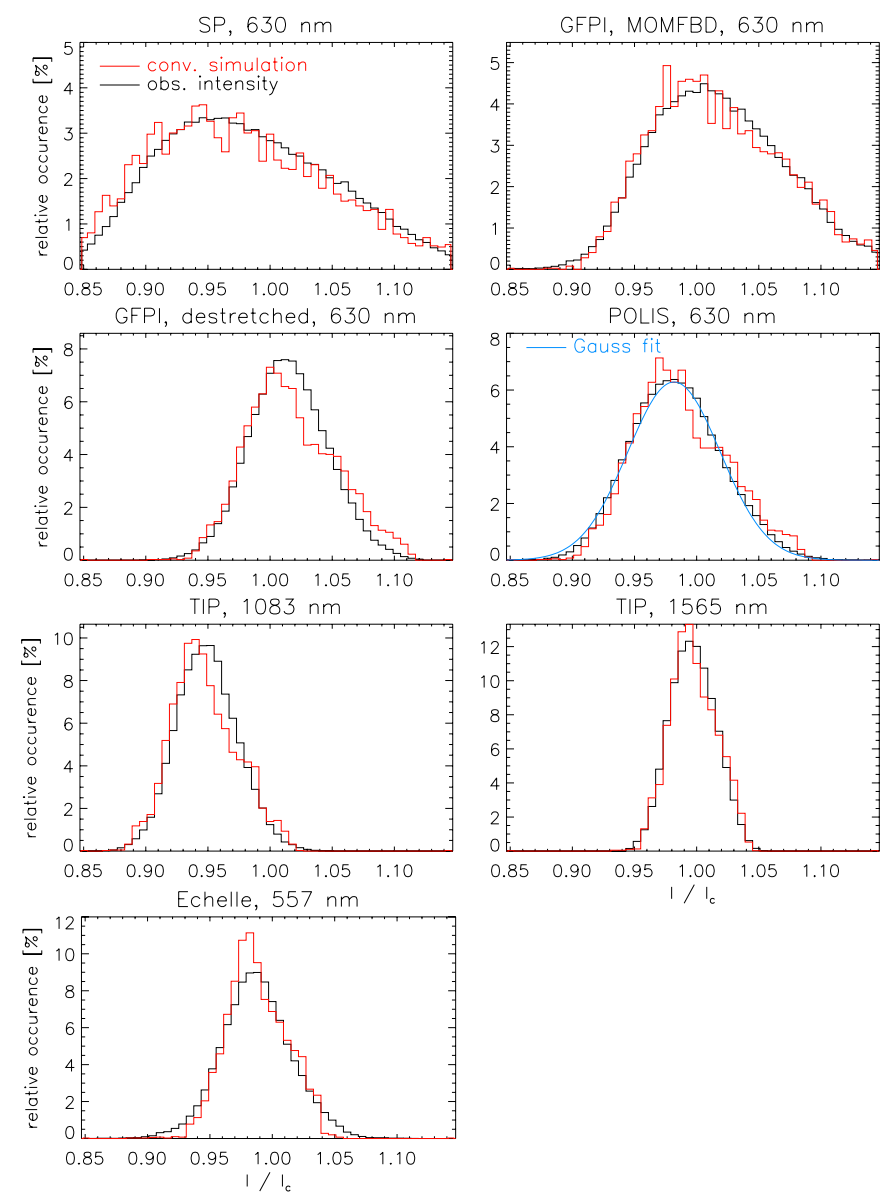

Fig. 6. Intensity histograms for the observations (black lines), and (red lines) for the HD simulation spatially convolved with the corresponding "best" kernel. Instrument and wavelength are given at the top of each panel. The blue line in the panel corresponding to the POLIS data denotes the Gaussian fit to the observed intensity distribution.

normalization of the spectra to the FTS atlas, because some of the chosen "continuum" wavelength ranges happened to fall in the far wings of strong lines (see Fig. 1). The rms contrasts were matched to $\sim 0.3 \%$ (cf. Table 4 below) and the intensity histograms of the observations are matched adequately.

The general match between observations and simulation throughout a two-dimensional (2D) FOV is shown in Fig. 7. We cut out a $6 \mathrm{Mm} \times 6 \mathrm{Mm}$ square from each observation for a visual comparison to the HD simulation convolved with the corresponding best kernel. The spatial patterns and the spatial resolution in $I_{\mathrm{c}}$ (top panel of Fig. 7), the velocity at $50 \%$ line depression (middle panel), and the line-core velocity (bottom panel) are well reproduced for all observations. Note, however, that the simulation data used for this comparison were already spectrally degraded as well (see the next section). Simulation data that were only spatially degraded showed some artifacts in the line-core velocity for all spectral lines except the low-forming Fe I lines at $1565 \mathrm{~nm}$. In case of the GFPI MOMFBD data (second column in Fig. 7) it would be impossible to distinguish the observed and synthetic maps if the corresponding labels were absent. The spatial resolution and the rms contrast of the shown section of the $557 \mathrm{~nm}$ data is higher than throughout the rest of the observed FOV at this wavelength because the part presented in the figure belongs to the region around the AO lock point. 
Table 3. Parameters of the spectral degradation from FTS/BASS atlas to observations.

\begin{tabular}{|c|c|c|c|c|c|c|c|c|c|c|c|c|}
\hline Instr. & \multicolumn{2}{|c|}{ SP } & \multirow{2}{*}{$\begin{array}{c}\text { GFPI, MOMFBD } \\
630.25\end{array}$} & \multirow{2}{*}{$\begin{array}{c}\text { GFPI, destr. } \\
630.25\end{array}$} & \multicolumn{2}{|c|}{$\overline{\text { POLIS }}$} & \multicolumn{3}{|c|}{$\overline{\text { TIP }}$} & \multicolumn{3}{|c|}{ Echelle } \\
\hline line & 630.15 & 630.25 & & & 630.15 & 630.25 & 1082.7 & 1564.8 & 1565.2 & 557.6 & 557.7 & 557.8 \\
\hline$\beta[\%]$ & 2.9 & 2.4 & 9.7 & 10.3 & 20.3 & 20.5 & 8.1 & 9.3 & 19.5 & 1.6 & 2.6 & 3.4 \\
\hline$\sigma^{\prime}[\mathrm{pm}]$ & 1.54 & 1.95 & 2.08 & 2.01 & 2.62 & 2.89 & 7.05 & 5.97 & 4.36 & 2.08 & 1.98 & 1.78 \\
\hline$\sigma^{\prime}\left[\mathrm{km} \mathrm{s}^{-1}\right]$ & 0.73 & 0.93 & 0.99 & 0.96 & 1.25 & 1.38 & 1.95 & 1.14 & 0.84 & 1.12 & 1.07 & 0.96 \\
\hline
\end{tabular}
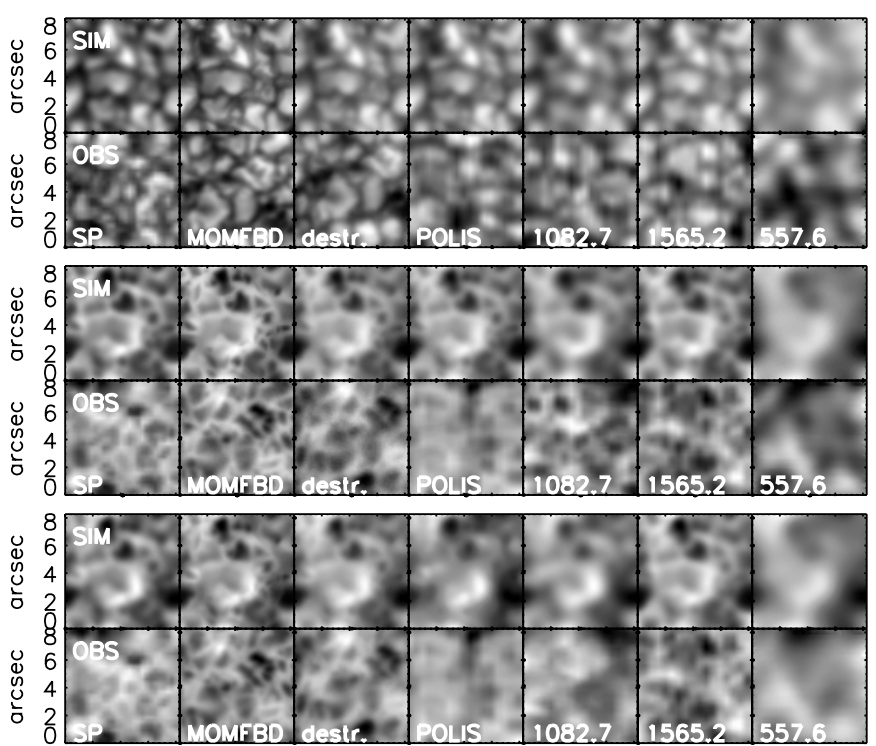

02468246824682468246824682468 arcsec arcsec arcsec arcsec arcsec arcsec arcsec

Fig. 7. Comparison of observations (lower row in each panel) with the HD simulation (upper row in each panel) convolved using each observation's "best" kernel. Top to bottom: $I_{\mathrm{c}}, v(50 \%$ line depression) and $v_{\text {core }}$ Left to right: SP, GFPI(MOMFBD), GFPI(destretched), POLIS, TIP@1083 nm, TIP@1565 nm and Echelle@557 nm.

\subsection{Spectral degradation}

The spectral degradation was determined by matching FTS or BASS atlas spectra and average observed profiles by adding a wavelength-independent stray-light offset $\beta$ to the atlas data and subsequently convolving them with a Gaussian $G$ of width $\sigma^{\prime}$ (Allende Prieto et al. 2004; Cabrera Solana et al. 2007) according to

$I_{\mathrm{FTS} / \mathrm{BASS}, \operatorname{degraded}}(\lambda)=\frac{I_{\mathrm{FTS} / \mathrm{BASS}}(\lambda)+\beta}{1+\beta} * G\left(\sigma^{\prime}, \lambda\right)$.

The value of $\beta$ only refers to spectral stray light produced by scattering inside the spectrometer, whereas the spatial stray light produced in front of the slit or the instrument entrance does not contribute to the spectral degradation (BE11). The squared deviation between the degraded FTS/BASS profile and the observed average profile as a function of $\beta$ and $\sigma^{\prime}$ yields again a matrix whose minimum indicates the best match of the profiles (e.g. Cabrera Solana et al. 2007, their Fig. A.1). We applied the procedure to all strong observed spectral lines in the respective wavelength regimes, in addition to those used for deriving the line parameters. The corresponding best-match parameters $\beta$ and $\sigma^{\prime}$ are listed in Table 3. For the spectral degradation of the simulations in those cases where multiple lines were observed with the same instrument and within the same spectral region (e.g. $1564.8 \mathrm{~nm}$ and $1565.2 \mathrm{~nm}$ ), we used the average values of $\beta$ and $\sigma^{\prime}$. The spectral stray-light levels range between $\sim 2 \%$ (Echelle, SP) and
$10 \%$ or more (TIP, POLIS). The velocity equivalents of the spectral broadenings are about $1 \mathrm{~km} \mathrm{~s}^{-1}$ in most cases.

After applying the spatial degradation, or spatial and spectral degradation to the synthetic spectra of the HD simulation (denoted in the following by HD-SPAT and HD-SPAT-SPEC, respectively), we determined the line parameters, and inverted the spectra with the settings described in the next section. The noise level in the observed intensity spectra is of the order of $10^{-3}$ of $I_{\mathrm{c}}$ and should have a negligible impact on the analysis results as long as only Stokes $I$ is involved. We therefore did not add noise to the synthetic spectra.

\subsection{Inversion setup}

Using the SIR code (Stokes Inversion based on Response functions; Ruiz Cobo \& del Toro Iniesta 1992), we inverted the spectra of all observations (SP, GFPI, POLIS, TIP), as well as the synthetic $630 \mathrm{~nm}$ spectra in the HD-FR, HD-SPAT and HDSPAT-SPEC. The spectral lines in our set of observations qualify as medium to strong lines and therefore provide information over an extended height range from the continuum forming layers up to $\log \tau \approx-1.5$ to -2 (cf. Allende Prieto et al. 2001; Cabrera Solana et al. 2005). The inversion was done with a fixed generic spatial stray-light level $\alpha$ of $20 \%$ for all the observations and for the HD-SPAT and HD-SPAT-SPEC cases, whereas $\alpha$ was set to zero for the inversion of the synthetic profiles at full resolution. Only one component (filling factor $100 \%$ ) was used in the inversion that was set to be either field-free or magnetic depending on whether the polarization degree in the spectra exceeded a specific threshold. This setup forced the inversion code to use just the temperature stratification to reproduce the observed Stokes $I$ profile on each pixel, and hence the temperature in the horizontal plane also follows the spatial intensity variation.

Inversion setups with a variable stray-light contribution or multiple inversion components do not reproduce the spatial variation of temperature well because of the interplay between temperature, stray-light contribution and/or filling factors (e.g. Martínez González et al. 2006). Effective magnetic filling factors are about $10 \%$ for ground-based observations at 1 " resolution (e.g. Martínez González et al. 2008; Beck \& Rezaei 2009) and reach $20 \%$ for SP data (Orozco Suárez et al. 2007). When using a variable stray-light contribution in a one-component inversion as the only factor taking the unresolved structure of the magnetic field into account, inversion codes will commonly tend to set it to unreasonably high (cf. Sect. 4.3) values of up to $80 \%$, because under such an assumption this factor has to reproduce both the real stray light and the magnetic filling factor inside a pixel (but see also Lagg et al. 2010; Martínez González et al. 2012). Our choice of the inversion setup yields an acceptable fit to the Stokes $I$ profiles (indicating a reasonable choice of $\alpha$ ) while the polarization signal in Stokes $Q U V$ is less well reproduced because of our one-component assumption. This has no impact on the current study because we do not make use of the retrieved information on the magnetic field parameters. 
Beck et al.: Thermodynamic fluctuations in photospheric convection simulations and observations
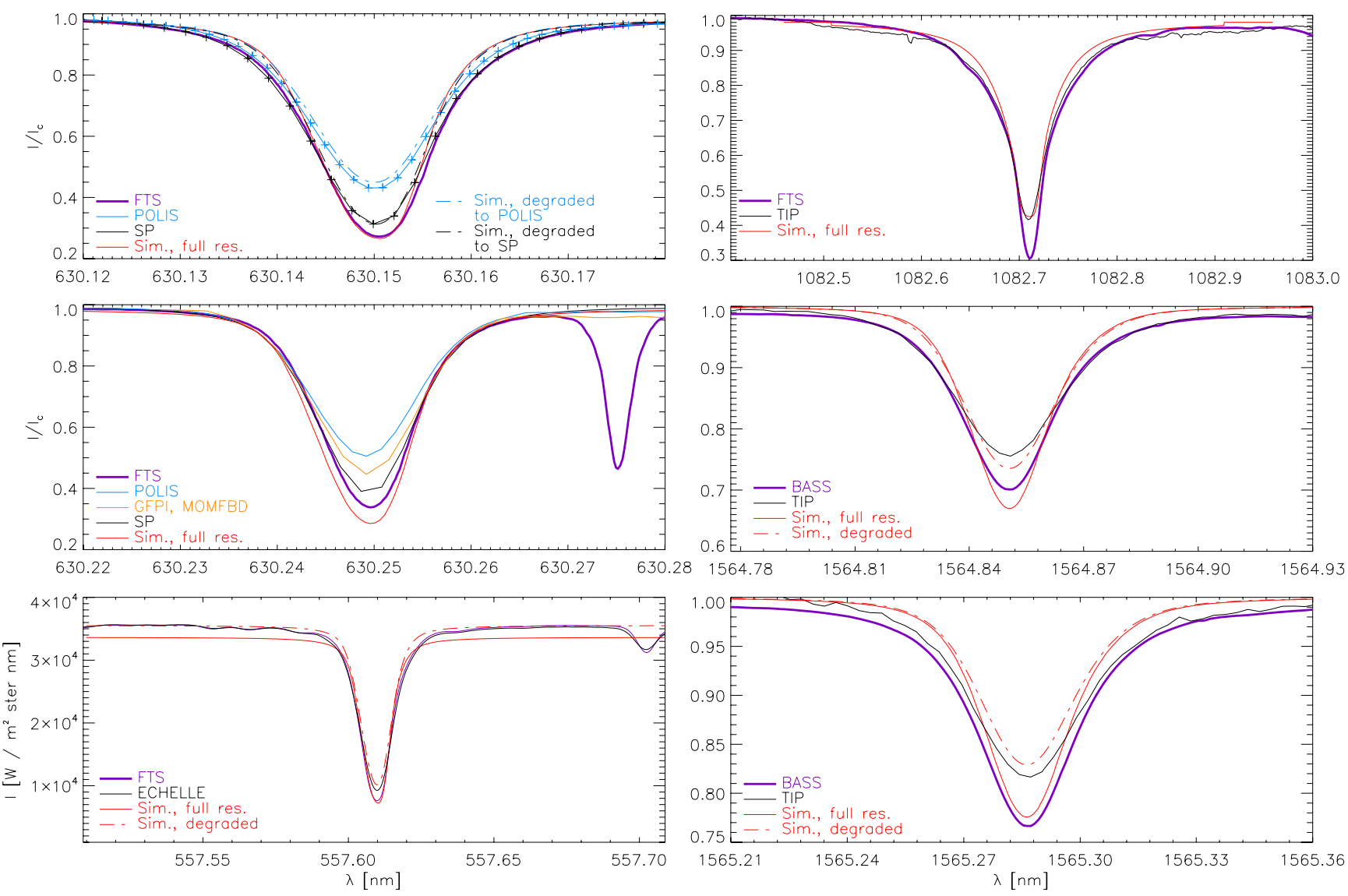

Fig. 8. Spatially averaged profiles of the $630 \mathrm{~nm}$ range (left top two rows), the $557 \mathrm{~nm}$ range (bottom left), and the near-IR wavelength ranges (right column). For the $630.15 \mathrm{~nm}$ line, the spectral sampling of the SP and POLIS is indicated by crosses. The thick purple curves show the spectra in the FTS or BASS atlas, depending on the wavelength range of interest. For $557.6 \mathrm{~nm}, 630.15 \mathrm{~nm}, 1564.8 \mathrm{~nm}$ and $1565.2 \mathrm{~nm}$ the profiles from the HD-SPAT-SPEC are over-plotted with dash-dotted lines. In the plot at $557.6 \mathrm{~nm}$, absolute flux units are used and the normalization of the HD-FR spectra to the FTS was undone.

We chose the Harvard Smithsonian Reference Atmosphere (HSRA; Gingerich et al. 1971) as initial temperature model. We used only two nodes (三variation of the initial model by linear gradients) for the temperature stratification. From the inversion, we retrieved $v_{\text {mac }}, v_{\text {mic }}$ and the temperature stratification $T$ as characteristic thermodynamic parameters.

\section{Results}

\subsection{Average line profiles of HD simulation and observations}

Figure 8 shows the spatially averaged profiles from the observations and from the HD simulation, as well as the atlas profiles. The average spectra from the HD-FR are expected to reflect the average thermodynamic conditions in the QS (even if with some limitations), and should thus provide a good fit to the observed FTS spectrum. Possible sources of deviation are the lack of magnetic broadening, a somewhat inadequate choice of thermodynamic parameters in the simulation and the transition parameters and solar abundances used in the spectral synthesis.

The comparison of the FTS or BASS profiles (thick purple curves in Fig. 8) with the HD-FR spectra (red lines) shows a good match for the $630.15 \mathrm{~nm}$ line, an acceptable match for $630.25 \mathrm{~nm}$ and from moderate to large deviations for the rest of the spectral lines, mainly because at the corresponding wavelengths (e.g. $557.6 \mathrm{~nm}, 1082.7 \mathrm{~nm}, 1565 \mathrm{~nm}$ ), the HDFR yields line profiles with narrower wings than present in the atlas spectra. This is likely due to the collisional broadening approximation employed in the spectral synthesis. For abundance determinations, spectral synthesis codes such as NICOLE (Socas-Navarro et al. 2000) can be employed that allow for the use of the more reliable collisional treatment developed by Anstee, Barklem, and O'Mara (ABO; see Barklem et al. 2000) as well as for the inclusion of non-LTE effects. The small deficiency $(\geqq 5 \%)$ in continuum intensity caused by the slightly too low effective temperature of the simulations (cf. Sect. 3) is demonstrated in the bottom left panel of Fig. 8. The normalization of all spectra to the atlas reference spectra removes it efficiently.

The spectral degradation determined in the previous section transforms the atlas spectra to the average profile of each observation. It therefore produces a good match of simulations and observations only when the spectra from the simulations at full resolution resemble the atlas spectra to start with. This is especially the case for the $630.15 \mathrm{~nm}$ line, but also for the rest of the lines the match between the degraded HD simulation and the observations is improved by the spectral degradation.

\subsection{Line parameters}

The spatial resolution of the HD simulation and the observations was matched using the continuum intensity (Figs. 6 and 7), whereas the spectral degradation was determined comparing average observed and atlas profiles without using the simulations' spectra. The comparison of the line parameters other than $I_{\mathrm{c}}$ therefore can reveal possible mismatches between the synthetic 


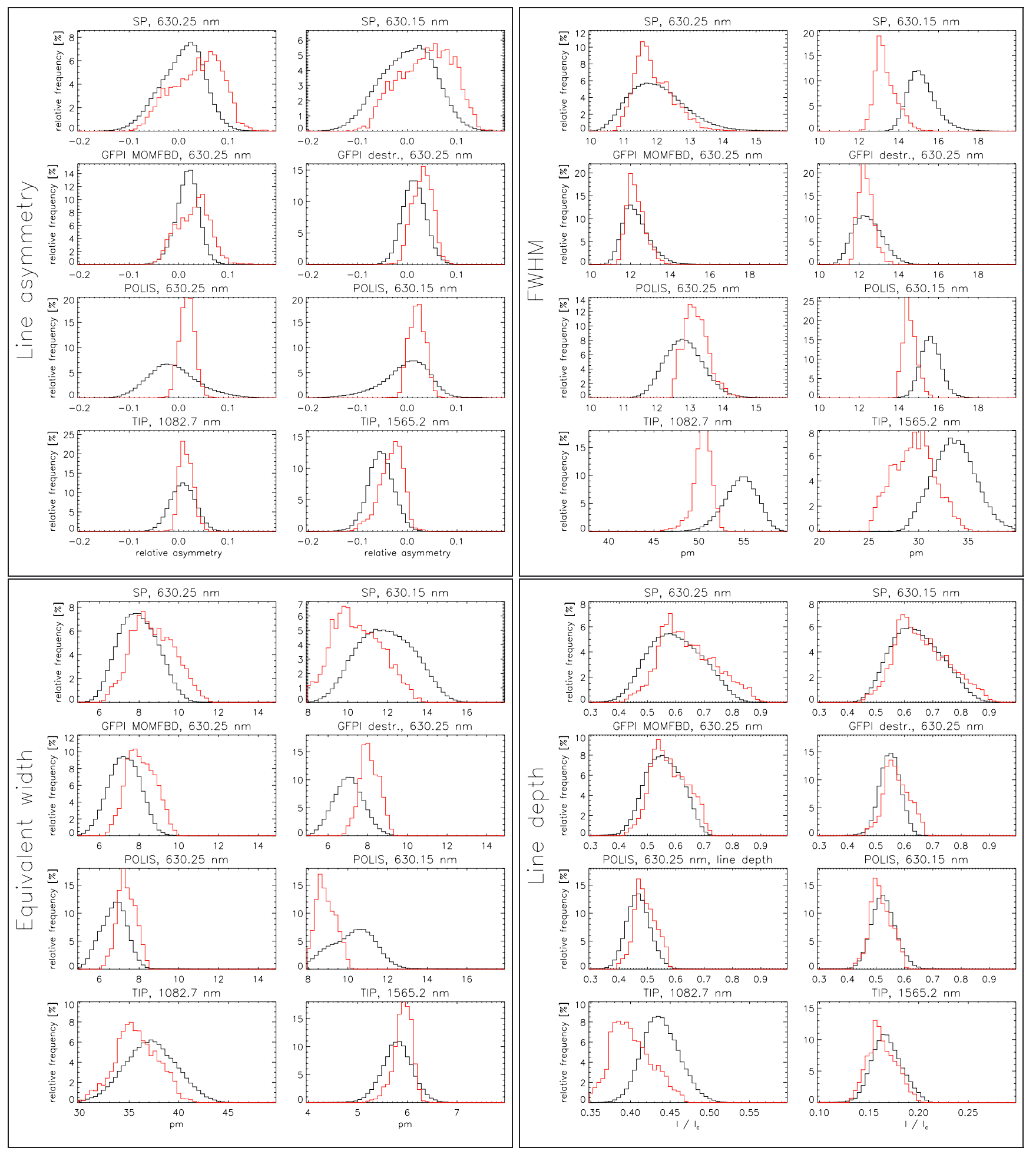

Fig. 9. Histograms of line parameters derived for HD-SPAT-SPEC and observations. Top left panel: line asymmetry. Top right panel: FWHM. Bottom left panel: equivalent width. Bottom right panel: line depth. The black line denotes the histogram of the relevant parameter for a given observational data set, the red line the one for the degraded simulation. Instrument and wavelength are denoted at the top of each panel.

and observed spectra that can indicate shortcomings of either simulations or observations (or both). Figure 9 shows the histograms of the line asymmetry, FWHM, equivalent width and line depth for the HD-SPAT-SPEC and for the observations. We note that the size of the statistical sample for observations and simulations differs by about one order of magnitude (cf. the bottom row in Table 1). For all of the following histograms, always the full FOV of the observations with several ten thousand profiles was used, whereas the simulations only provide about 4000 profiles.

The line asymmetry (top left panel in Fig. 9) is seen to be sensitive to the spatial resolution, as also found by PuB11. The shape of the line asymmetry histogram in both observations and simulations changes from a roughly Gaussian distribution at the spatial resolution of the destretched GFPI data (second column, second row) to a broad, flatter distribution with a tail towards 
Table 4. Line parameters in observations and simulations.

\begin{tabular}{|c|c|c|c|c|c|c|c|c|}
\hline $\begin{array}{l}\text { Type } \\
\text { units }\end{array}$ & $\begin{array}{c}\text { Line } \\
\mathrm{nm}\end{array}$ & $\begin{array}{c}\mathrm{rms} I_{\mathrm{c}} \\
\%\end{array}$ & $\begin{array}{c}\operatorname{rms} v(50 \%) \\
\mathrm{m} \mathrm{s}^{-1}\end{array}$ & $\begin{array}{c}\mathrm{rms} v_{\text {core }} \\
\mathrm{m} \mathrm{s}^{-1}\end{array}$ & $\begin{array}{c}\text { Line depth } \\
\left\langle I_{\mathrm{c}}\right\rangle\end{array}$ & $\begin{array}{c}\text { Core intensity } \\
\left\langle I_{\mathrm{c}}\right\rangle\end{array}$ & $\begin{array}{c}\text { Asymmetry } \\
\delta A\end{array}$ & $\begin{array}{l}\text { Eq. width } \\
\text { pm }\end{array}$ \\
\hline \multicolumn{9}{|c|}{ ECHELLE } \\
\hline HD FULL RES & 557.6 & 18.1 & 890 & 968 & $0.830 \pm 0.225$ & $0.203 \pm 0.032$ & $-0.033 \pm 0.229$ & $9.71 \pm 2.80$ \\
\hline HD SPAT & 557.6 & 2.4 & 247 & 173 & $0.764 \pm 0.035$ & $0.215 \pm 0.009$ & $0.099 \pm 0.031$ & $9.65 \pm 0.35$ \\
\hline HD SPAT+SPEC & 557.6 & 2.4 & 234 & 227 & $0.689 \pm 0.030$ & $0.281 \pm 0.012$ & $0.036 \pm 0.012$ & $9.39 \pm 0.34$ \\
\hline $\mathrm{OBS}^{1}$ & 557.6 & 2.6 & 282 & 316 & $0.679 \pm 0.033$ & $0.257 \pm 0.016$ & $0.020 \pm 0.027$ & $12.27 \pm 0.43$ \\
\hline \multicolumn{9}{|c|}{ POLIS } \\
\hline HD FULL RES & 630.15 & 15.2 & 1102 & 986 & $0.760 \pm 0.190$ & $0.254 \pm 0.034$ & $-0.032 \pm 0.211$ & $10.76 \pm 2.72$ \\
\hline HD SPAT & 630.15 & 3.7 & 362 & 224 & $0.704 \pm 0.052$ & $0.266 \pm 0.012$ & $0.091 \pm 0.052$ & $10.72 \pm 0.63$ \\
\hline HD SPAT+SPEC & 630.15 & 3.7 & 288 & 315 & $0.523 \pm 0.037$ & $0.450 \pm 0.013$ & $0.023 \pm 0.016$ & $8.95 \pm 0.53$ \\
\hline OBS & 630.15 & 3.7 & 392 & 358 & $0.530 \pm 0.059$ & $0.425 \pm 0.034$ & $-0.006 \pm 0.048$ & $10.23 \pm 1.57$ \\
\hline HD FULL RES & 630.25 & 15 & 1097 & 821 & $0.756 \pm 0.201$ & $0.269 \pm 0.049$ & $-0.009 \pm 0.225$ & $8.98 \pm 2.35$ \\
\hline HD SPAT & 630.25 & 3.7 & 365 & 231 & $0.688 \pm 0.056$ & $0.285 \pm 0.018$ & $0.084 \pm 0.050$ & $8.94 \pm 0.54$ \\
\hline HD SPAT+SPEC & 630.25 & 3.7 & 474 & 322 & $0.498 \pm 0.038$ & $0.481 \pm 0.015$ & $0.021 \pm 0.015$ & $7.46 \pm 0.45$ \\
\hline OBS & 630.25 & 3.7 & 381 & 353 & $0.472 \pm 0.056$ & $0.503 \pm 0.038$ & $-0.007 \pm 0.050$ & $6.76 \pm 0.93$ \\
\hline \multicolumn{9}{|c|}{ SP } \\
\hline HD SPAT & 630.15 & 7.2 & 594 & 360 & $0.717 \pm 0.098$ & $0.262 \pm 0.019$ & $0.087 \pm 0.103$ & $10.74 \pm 1.24$ \\
\hline HD SPAT+SPEC & 630.15 & 7.2 & 455 & 464 & $0.670 \pm 0.093$ & $0.307 \pm 0.023$ & $0.041 \pm 0.053$ & $10.57 \pm 1.24$ \\
\hline OBS & 630.15 & 6.9 & 619 & 498 & $0.650 \pm 0.087$ & $0.307 \pm 0.034$ & $0.004 \pm 0.055$ & $11.99 \pm 1.40$ \\
\hline HD SPAT & 630.25 & $\overline{7.2}$ & 591 & 373 & $0.703 \pm 0.105$ & $0.280 \pm 0.028$ & $0.080 \pm 0.096$ & $8.96 \pm 1.07$ \\
\hline HD SPAT+SPEC & 630.25 & 7.2 & 607 & 483 & $0.649 \pm 0.097$ & $0.333 \pm 0.030$ & $0.040 \pm 0.049$ & $8.81 \pm 1.06$ \\
\hline OBS & 630.25 & 6.9 & 614 & 537 & $0.606 \pm 0.094$ & $0.374 \pm 0.040$ & $0.011 \pm 0.044$ & $8.06 \pm 0.97$ \\
\hline \multicolumn{9}{|c|}{ GFPI MOMFBD } \\
\hline HD SPAT & 630.25 & 5.3 & 474 & 271 & $0.693 \pm 0.076$ & $0.283 \pm 0.022$ & $0.081 \pm 0.074$ & $8.94 \pm 0.78$ \\
\hline HD SPAT+SPEC & 630.25 & 5.3 & 511 & 379 & $0.583 \pm 0.063$ & $0.396 \pm 0.022$ & $0.033 \pm 0.032$ & $8.20 \pm 0.72$ \\
\hline OBS & 630.25 & 5.3 & 443 & 439 & $0.564 \pm 0.064$ & $0.460 \pm 0.031$ & $0.022 \pm 0.023$ & $7.36 \pm 0.79$ \\
\hline \multicolumn{9}{|c|}{ GFPI destretched } \\
\hline HD SPAT & 630.25 & 3.7 & 356 & 224 & $0.701 \pm 0.055$ & $0.291 \pm 0.018$ & $0.083 \pm 0.048$ & $9.11 \pm 0.54$ \\
\hline HD SPAT+SPEC & 630.25 & 3.7 & 474 & 298 & $0.579 \pm 0.044$ & $0.399 \pm 0.017$ & $0.034 \pm 0.020$ & $8.19 \pm 0.48$ \\
\hline OBS & 630.25 & 3.2 & 283 & 300 & $0.556 \pm 0.037$ & $0.464 \pm 0.022$ & $0.018 \pm 0.023$ & $7.13 \pm 0.74$ \\
\hline \multicolumn{9}{|c|}{$\overline{T I P}$} \\
\hline HD FULL RES & 1082.7 & 9.0 & 1246 & 1539 & $0.484 \pm 0.084$ & $0.427 \pm 0.016$ & $-0.024 \pm 0.125$ & $39.76 \pm 8.38$ \\
\hline HD SPAT & 1082.7 & 2.7 & 413 & 517 & $0.475 \pm 0.030$ & $0.433 \pm 0.003$ & $0.076 \pm 0.061$ & $39.67 \pm 2.35$ \\
\hline HD SPAT+SPEC & 1082.7 & 2.7 & 405 & 357 & $0.403 \pm 0.026$ & $0.493 \pm 0.006$ & $0.019 \pm 0.013$ & $35.93 \pm 2.13$ \\
\hline OBS & 1082.7 & 2.5 & 398 & 415 & $0.463 \pm 0.024$ & $0.438 \pm 0.014$ & $0.045 \pm 0.025$ & $38.03 \pm 2.66$ \\
\hline HD FULL RES & 1564.8 & 7.9 & 1347 & 1118 & $0.397 \pm 0.093$ & $0.603 \pm 0.042$ & $0.001 \pm 0.167$ & $9.57 \pm 1.58$ \\
\hline HD SPAT & 1564.8 & 1.9 & 554 & 378 & $0.318 \pm 0.031$ & $0.666 \pm 0.022$ & $-0.016 \pm 0.030$ & $9.53 \pm 0.32$ \\
\hline HD SPAT+SPEC & 1564.8 & 1.9 & 347 & 385 & $0.259 \pm 0.021$ & $0.731 \pm 0.015$ & $-0.025 \pm 0.020$ & $8.71 \pm 0.29$ \\
\hline OBS & 1564.8 & 1.8 & 397 & 431 & $0.227 \pm 0.021$ & $0.760 \pm 0.017$ & $-0.033 \pm 0.027$ & $8.87 \pm 0.36$ \\
\hline HD FULL RES & 1565.2 & 7.9 & 1453 & 1292 & $0.273 \pm 0.060$ & $0.718 \pm 0.041$ & $0.005 \pm 0.137$ & $7.15 \pm 1.06$ \\
\hline HD SPAT & 1565.2 & 1.9 & 563 & 426 & $0.213 \pm 0.021$ & $0.772 \pm 0.018$ & $-0.027 \pm 0.032$ & $7.13 \pm 0.22$ \\
\hline HD SPAT+SPEC & 1565.2 & 1.9 & 367 & 414 & $0.165 \pm 0.014$ & $0.826 \pm 0.013$ & $-0.029 \pm 0.024$ & $5.94 \pm 0.18$ \\
\hline OBS & 1565.2 & 1.8 & 405 & 445 & $0.170 \pm 0.015$ & $0.822 \pm 0.016$ & $-0.050 \pm 0.027$ & $5.83 \pm 0.31$ \\
\hline \multicolumn{9}{|c|}{ Simulation, $\mathrm{MHD}^{2}$, full resolution } \\
\hline MHD FULL RES & $630.25,50 \mathrm{G}$ & 14.0 & 1067 & 856 & $0.740 \pm 0.192$ & $0.283 \pm 0.088$ & $-0.023 \pm 0.214$ & $8.95 \pm 2.19$ \\
\hline MHD FULL RES & $630.25,100 \mathrm{G}$ & 14.1 & 1058 & 909 & $0.716 \pm 0.207$ & $0.305 \pm 0.116$ & $-0.023 \pm 0.214$ & $8.80 \pm 2.23$ \\
\hline MHD FULL RES & $630.25,200 \mathrm{G}$ & 13.8 & 1047 & 968 & $0.679 \pm 0.216$ & $0.339 \pm 0.140$ & $-0.001 \pm 0.199$ & $8.70 \pm 2.06$ \\
\hline
\end{tabular}

Notes. ${ }^{(1)}$ OBS: observations; ${ }^{(2)}$ MHD-FR with average magnetic flux of 50, 100, 200 G, respectively.

negative asymmetries at the resolution of the SP (top row). The line asymmetries in the observed POLIS spectra show a much broader histogram with significantly higher absolute values than the HD-SPAT-SPEC, which is also the case for most of the other line parameters from POLIS in Fig. 9 in one or both $630 \mathrm{~nm}$ lines. We suspect the presence of some spectral degradation effect that varies along the slit such as a varying spectral focus as a reason, because the average profiles matched very well (Fig. 8). The line depth in the data set used is also somewhat lower than for other POLIS observations (cf. Beck et al. 2005b, their Fig. 6). The line asymmetries of the near-IR lines at $1565 \mathrm{~nm}$ are smaller than those of all other lines because of the smaller formation height ranges of the former.
The shapes of the histograms of the FWHM (top right panel) and equivalent width (bottom left panel) are usually similar for the HD-SPAT-SPEC and the observations, but in some cases the histograms are (slightly) displaced from each other. There is a slight tendency for the HD-SPAT-SPEC to have a lower FWHM, but there are also reverse cases. A better match of the FWHM or equivalent width would require some fine-tuning in their determination (e.g. in the case of equivalent width measurements, accounting for line blends) and in the synthesis setup (e.g. abundances, line broadening). This issue, however, should not have a critical impact on the spatial distribution of the values caused by the thermodynamics. The line depth (bottom-right panel) shows a very good match for all lines, except for the 

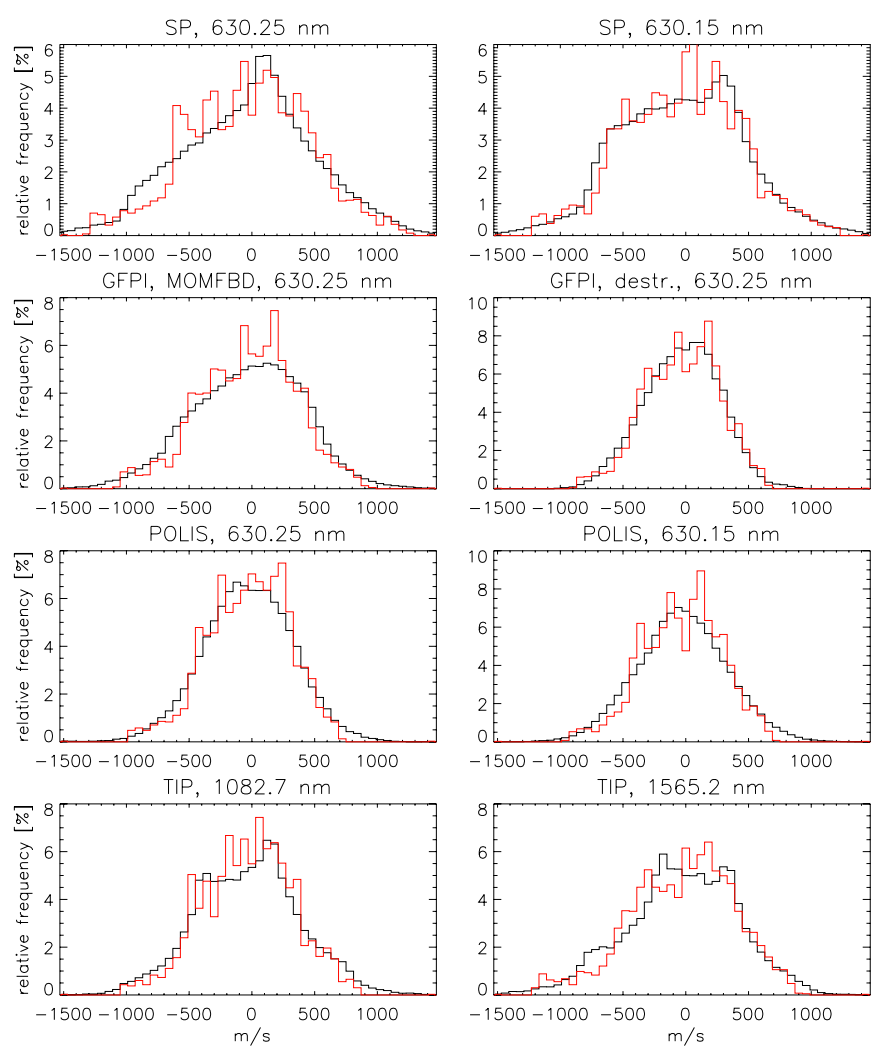

Fig. 10. Histograms of line-core velocity in HD-SPAT-SPEC and observations. The black line denotes the histogram of the parameter in the observation and the red line that in the HD-SPAT-SPEC. Instrument and central wavelength of relevance are specified in the title of each sub-panel.

Si I line at $1082.7 \mathrm{~nm}$. All histograms share a common trend with increasing spatial resolution, i.e. they become more asymmetric/skewed rather than only becoming broader. Figure B.1 shows the corresponding histograms of the Fe I line at $557.6 \mathrm{~nm}$ for completeness.

The histograms of the line-core velocity are shown in Fig. 10 (see Fig. B.1 for $557.6 \mathrm{~nm}$ ). The line-core velocity reflects the dynamics of the mass motions in the upper end of the respective formation heights of the various spectral lines. The match between the HD simulation and the observations is excellent, nearly as good as the one found for the continuum intensity distributions (Fig. 6) that we employed for the determination of the spatial degradation. For the SP data in $630.15 \mathrm{~nm}$ and the nearIR data in $1082.7 \mathrm{~nm}$ and $1565.2 \mathrm{~nm}$, the line-core velocity histograms for the observational data show a peculiar shape, with a central plateau at low velocities, a steep decline at intermediate velocities and a low probability of finding velocities above about $\pm 500 \mathrm{~m} \mathrm{~s}^{-1}$. This shape turns out to be reproduced surprisingly well by the HD-SPAT-SPEC and appears also in other convection simulations (e.g. Stein \& Nordlund 1998; Beeck et al. 2012).

Table 4 lists the average and rms values of some line parameters for the observations, the HD-FR, the HD-SPAT and the HDSPAT-SPEC. We also included the parameters of the $630.25 \mathrm{~nm}$ line in the MHD simulations with increasing average magnetic flux $(50-200 \mathrm{G})$ at full resolution for comparison (lowermost three rows in Table 4).

The clearest trend in the observational values is the increase in all rms fluctuations with increasing spatial resolution, yielding a factor of about 1.5 between, e.g., POLIS and the SP in the rms velocities (fourth column of Table 4). The HD-FR shows rms velocities (compare the values with, e.g., Asplund et al. 2004, last column of their Table 1) higher by an additional factor of about two in comparison to SP and GFPI observations. Whereas the spatial degradation of the simulations always significantly decreases the corresponding rms fluctuations, the spectral degradation has in some cases the opposite effect of increasing the rms values, although only slightly (i.e. spectral degradation has a weaker impact than spatial degradation). The observed rms velocities are usually matched in the HD-SPAT-SPEC to within about $\pm 50 \mathrm{~m} \mathrm{~s}^{-1}$. In the case of the line-core velocity $v_{\text {core }}$, the rms in the HD-SPAT-SPEC is lower than in the observations for all lines considered.

The line depth (sixth column of Table 4) of the HD simulation is usually reduced only slightly by the spatial degradation and more strongly by the spectral degradation, whereas the linecore intensity (seventh column of Table 4) naturally shows a corresponding increase. The factor with the biggest impact on the changes in line depth and line-core intensity is the stray-light offset $\beta$ in the spectral degradation. The significant consequences of these changes in the line shape on the temperature stratifications retrieved by the inversion are discussed in Sect. 5.4 below.

The average line asymmetry (last-but-one column in Table 4) changes strongly with both the spatial or spectral degradation of the simulations, even including a change in sign. In most cases, the change from positive to negative asymmetry (or reverse) by the degradation is actually necessary to match the observed value (e.g. for $557.6 \mathrm{~nm}$, for $630 \mathrm{~nm}$ in the SP and GFPI data, and for all near-IR lines). Assuming the same temperature structure in granules and intergranular lanes (IGLs), decelerating upflows (accelerating downflows) lead to a positive (negative) line asymmetry. The spatial average of the line asymmetry measures an intensity-weighted relative area filling factor of granules and small-scale IGLs, which, after the latter are largely suppressed by the spatial degradation process, yields mainly positive values.

Accounting for spatial degradation reduces the equivalent width (last column in Table 4) of the spectral lines by 0.02-0.09 pm, depending on the line, except for the destretched GFPI data at $630.25 \mathrm{~nm}$, for which it increases the equivalent width by $0.13 \mathrm{pm}$. The increase in magnetic flux in the MHDFR reduces the equivalent width of the $630.25 \mathrm{~nm}$ line by up to $\sim 0.3 \mathrm{pm}$ (HD-200 G). This effect is due to the warmer atmospheric temperature structure generally retrieved in the presence of magnetic fields that causes line-core weakening (line-gap; e.g. Stellmacher \& Wiehr 1971; Chapman 1977; Hirzberger \& Wiehr 2005). The corresponding decrease in equivalent width is the dominant effect of the presence of magnetic fields on iron lines in the visible wavelength range, even if they are also Zeemansensitive. At visible wavelengths, the Zeeman broadening is not sufficiently strong to counter the indirect effect on spectral lines due to the change in average temperature induced by magnetic flux (Fabbian et al. 2010). The spectral degradation tends to reduce the equivalent width by even larger amounts (up to several pm for the $1082.7 \mathrm{~nm}$ line), because the spectral stray-light offset $\beta$ (cf. Table 3 ) directly scales the line depth, and hence also the total area of the absorption profile.

\subsection{Line properties at different line depression levels}

The bisector is defined as the curve connecting the central positions between left and right wing at different line depression levels throughout an absorption line. We determined the bisector position and the line width at ten different intensity levels throughout the absorption profiles. The variation of these 
Beck et al.: Thermodynamic fluctuations in photospheric convection simulations and observations

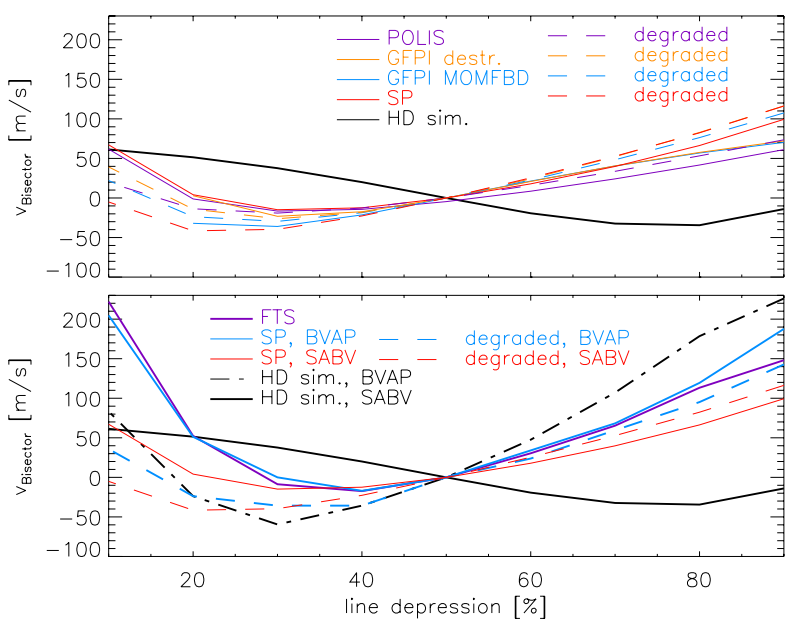

Fig. 11. Top panel: spatially averaged bisector velocity (SABV) for the $630.25 \mathrm{~nm}$ line in the observations (solid lines), the HD-FR (solid black line) and the HD-SPAT-SPEC (dashed lines). Bottom panel: comparison between SABVs and bisector velocities of average profiles (BVAP) for the $630.25 \mathrm{~nm}$ line. Solid/dash-dotted black lines: SABV/BVAP in the HD-FR. Solid red/blue lines: SABV/BVAP for the SP data. Red/blue dashed lines: SABV/BVAP for the simulation degraded to SP resolution. The purple line gives the bisector in the FTS atlas profile. Positive velocities correspond to redshifts relative to the average velocity at $50 \%$ line depression.

properties in an individual spectral line profile traces the values of the corresponding thermodynamic stratification in the atmosphere and any possible gradients. A line depression of $0 \%$ denotes the continuum level, whereas $100 \%$ denotes the line core. We used the average bisector position at $50 \%$ line depression as zero point reference for the conversion to velocities.

The bisector positions at different line depression levels trace gradients of velocity with optical depth. For average spectra in QS granulation, the bisectors usually yield a C-shape throughout the different levels of line depression (e.g. Brandt \& Schroeter 1982; Dravins 1982, 2008; Marquez et al. 1996; Puschmann et al. 1999; Hanslmeier et al. 2000; Asplund et al. 2000; Mikurda et al. 2006; Gray 2010; de la Cruz Rodríguez et al. 2011). The upper panel of Fig. 11 shows the spatial average of the bisector positions, converted to the corresponding velocities, for the $630.25 \mathrm{~nm}$ line in the observations and the HD simulation. A prominent difference is seen: the averaged bisector velocity of the HD-FR has a roughly inverse shape to that of all observations. The match between the velocity at different line depression levels in observed and synthetic spectra is, however, good for the spatially and spectrally degraded HD simulation, except for the lines at $557.6 \mathrm{~nm}$ and $1082.7 \mathrm{~nm}$ (Fig. A.1). For $557.6 \mathrm{~nm}$, the deviations at line depression levels below $40 \%$ are presumably caused by the weak blends in its line wing. Note that the spatial average of the bisector velocities does not reflect the dynamical range of the vertical velocity fluctuations, but rather the average net vertical flow speed.

To determine the reason for the inversion of the bisector shape between observations and the HD-FR, we reversed the order of the bisector determination and the spatial averaging by calculating also bisectors for spatially averaged profiles. The lower panel of Fig. 11 compares the spatially averaged bisector velocities (SABV) and the bisector velocities of average profiles (BVAP) for the $630.25 \mathrm{~nm}$ line. We included the SP data, the HD-FR, the HD simulation spatially and spectrally degraded to the SP data, and the profile of the FTS atlas. Applying the spatial averaging to the spectra before determining the bisectors flips the shape of the bisector velocities with respect to the HD-FR (compare the black solid and black dash-dotted lines in the lower panel of Fig. 11). The similarity of the BVAP for the simulation (black dash-dotted line) and the SABV for the HD simulation degraded to SP resolution (red dashed line), or the SP data themselves (red solid line), indicates that the change in the shape must be introduced by a small-scale averaging between the full spatial sampling of the simulation $\left(0{ }^{\prime} 13 \sim 94 \mathrm{~km}\right)$ and the resolution of the SP data $\left(0 .^{\prime} 62 \sim 450 \mathrm{~km}\right)$. The BVAP of the HD-FR also matches the bisector shape of the MOMFBD GFPI data (blue line in the upper panel of Fig. 11), which puts an even stricter upper limit to the spatial averaging effect of about $0.3(\sim 218 \mathrm{~km})$. The reversal of the shape of the bisector should also correspond to the sign change in the line asymmetry by the spatial degradation that was found before (cf. Sect. 5.2 and Table 4) and should be coupled to the relative area fraction of bright granules and dark intergranular lanes.

Compared to the small-scale spatial average caused already by the effective spatial resolution of the observations, any additional large-scale averaging done in the BVAP process adds only minor modifications. The SABV and BVAP of the SP data are similar (albeit not fully identical), and the bisector from the FTS atlas profile matches the BVAP from the SP data well for the $630.25 \mathrm{~nm}$ line. These two facts are indicative of two different subsequent averaging effects: on the one hand, the spatial averaging caused by the limited spatial resolution of all of our observations has already significantly changed the shape of the bisector velocity curve in comparison to the HD-FR. On the other hand, averaging over the full FOV (or a long time) leads to line profiles, and hence bisectors similar to FTS regardless of the spatial resolution of the input spectra. This second averaging step thus only produces minor variations of the bisector shape.

In contrast to the average position of the bisectors, the rms fluctuations of the bisector velocities do reflect the vertical velocity fluctuations caused by the dynamical evolution of the solar atmosphere. For the SP observations both at $630.25 \mathrm{~nm}$ (top panel of Fig. 12) and at $630.15 \mathrm{~nm}$ (Fig. A.4) and for the nearIR observations (Fig. A.4), an increase in the velocity rms is seen as one moves from the higher atmospheric levels towards the continuum formation layers in the lower atmosphere, i.e. for high bisector levels (line depression levels $<40 \%$ ). In the observed SP data at $630 \mathrm{~nm}$, the velocity rms decreases monotonically with increasing line depression, like also in the HD-FR for all spectral lines. Towards low line depression levels $(<40 \%)$ in most lines, the degraded HD simulation shows lower rms fluctuations than the observations, whereas the rms values are nearly identical near the line-core. The bisector velocity rms for the $557.6 \mathrm{~nm}$ line is shown in Fig. B.2 for completeness.

The decrease in the rms velocity fluctuations with height in the atmosphere indicates that the velocity formation heights for most of the spectral lines do not reach into the realm where propagating acoustic waves of increasing amplitude dominate the atmosphere making the rms velocities increase again strongly with height (e.g. Beck et al. 2009). The spectral lines used here therefore in general correspond to an height regime that is dominated by the convective velocity distribution as far as the line-of-sight (LOS) velocities are concerned.

The bisector intensity rms shows a different height dependence (e.g. middle panel of Fig. 12) than the bisector velocity rms. The relative (i.e. normalized by the average intensity) rms fluctuations of the intensity levels show increased fluctuations for line depression levels above about $70 \%$. This indicates an increase in temperature fluctuations close to the line core, as expected for propagating acoustic waves with a steepening wave 


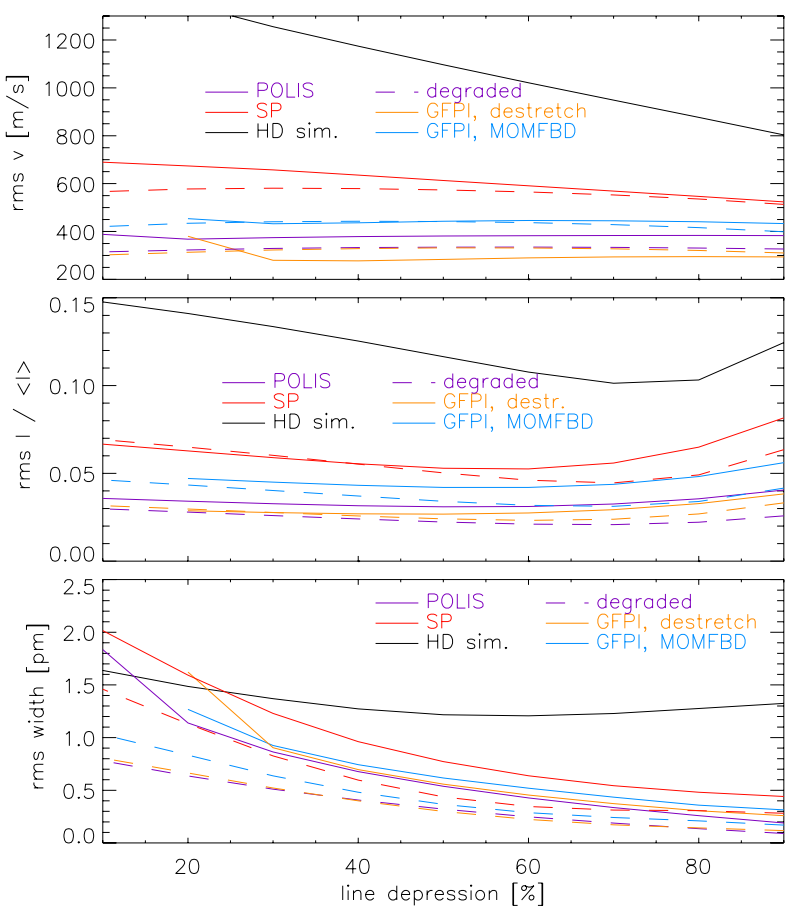

Fig. 12. Rms fluctuations in the line properties at different line depression levels for the $630.25 \mathrm{~nm}$ line in the observations (solid lines), in the HD-FR (solid black line) and in the HD-SPAT-SPEC (dashed lines). Top: bisector velocity. Middle: bisector intensity. Bottom: line width.

amplitude. This explanation stands at least for the HD simulation that is free of magnetic flux, whereas in the observations it could also in principle be related to the presence of magnetic fields, because the latter lead to brightenings in the line core (cf. Puschmann et al. 2007, or Fig. 2, middle row: the brightenings in the lower left corner of the FOV for the GFPI data are of a magnetic origin (see PuB11)). The rms of the intensity in the observations generally exceeds the fluctuations in the HDSPAT-SPEC.

The rms fluctuations in the line width at different line depression levels for $630.25 \mathrm{~nm}$ are shown in the bottom panel of Fig. 12. In this case, the rms fluctuations start to increase when reaching about $60 \%$ in line depression for the $630 \mathrm{~nm}$ lines (as well as for the $557.6 \mathrm{~nm}$ line, Fig. B.2) in the HD-FR, whereas all observations and the HD-SPAT-SPEC show a monotonic decrease in the rms with increasing line depression. The fluctuations of all parameters in the spatially degraded HD simulation generally are smaller than those in the observations besides for the low-forming $1565 \mathrm{~nm}$ lines, where the HD-SPAT-SPEC match the observations nearly perfectly (Fig. A.4). The average values of the bisector intensity and the line width at different line depression levels are shown in Figs. A.2 and A.3 for completeness.

\subsection{Inversion results}

In the inversion, the macroturbulent velocity $v_{\text {mac }}$ is used to convolve the synthetic profiles with a Gaussian of variable width to match the observed spectra. The microturbulent velocity $v_{\text {mic }}$ is one of the contributions to the line width in a Lorentzian function that is used in the integration of the radiative transfer equation (see, e.g., Rutten 2003). The value of $v_{\text {mic }}$ could be specified to vary on the optical depth scale in the SIR code, but we set it to be constant at all $\tau$ values. The two ad hoc empirical parameters $v_{\text {mac }}$ and $v_{\text {mic }}$ encompass two different physical effects when observed spectra are inverted: the "real" macro/microturbulence caused by unresolved macro/microscopic velocities in the solar atmosphere and the spectral broadening caused by the characteristics of the respective spectrometer. While the former component should scale with the spatial resolution, the latter should be a constant characteristic of the spectrometer that in some inversion codes can be included by providing a measurement of the PSF such as those in Sect. 4.4. In the case of the simulations without any degradation, both effects are absent; for the spatially degraded simulations, only the broadening by unresolved velocities is included.

The top row of Fig. 13 shows the histograms of the macroturbulent (left column) and microturbulent (right column) velocities derived by applying the SIR code for the inversion of the different observed spectra. Only one value per wavelength range was derived. Even when multiple spectral lines were present in a given range, they were in fact inverted simultaneously. The middle and bottom row of Fig. 13 show the macroturbulent and microturbulent velocities retrieved from inverting the HD-SPAT and HD-SPAT-SPEC, respectively. The values from inverting the HD-FR are overplotted with black lines in each panel as reference.

Macroturbulent velocity There is an offset in macroturbulent velocity between the observations at $630 \mathrm{~nm}$ (most $v_{\text {mac }}<$ $2 \mathrm{~km} \mathrm{~s}^{-1}$ ) and in the near-IR (most $v_{\mathrm{mac}}>2 \mathrm{~km} \mathrm{~s}^{-1}$ ) that is presumably caused by the lower spectral sampling and/or resolution of the near-IR observations with TIP (top left panel of Fig. 13). The distribution of $v_{\mathrm{mac}}$ for the $1082.7 \mathrm{~nm}$ line is significantly broader than those obtained for any of the other observations. This was found to result from using only two nodes in the temperature stratification. An inversion of the observation in this line using three nodes yielded a distribution of $v_{\text {mac }}$ that was similar to that of all other observations, with a much smaller width and a roughly Gaussian shape. The Si I line at $1082.7 \mathrm{~nm}$ has an extended formation height and is sensitive to NLTE effects (cf. Shchukina et al. 2012, and references therein).

For all $630 \mathrm{~nm}$ observations, a slight trend with spatial resolution can be seen, with POLIS yielding the highest and the GFPI the lowest average $v_{\text {mac }}\left(1.7-1.9 \mathrm{~km} \mathrm{~s}^{-1}\right.$, cf. Table 5). For the observational data, the spatial structuring in $2 \mathrm{D}$ maps of $v_{\text {mac }}$ roughly mirrored an inverse granulation pattern, with increased $v_{\text {mac }}$ in intergranular lanes, especially on all locations where polarization signal was also present. Such a characteristic resulting pattern is likely related to the one-component inversion setup, where the missing freedom in the magnetic fill factor forced the inversion code to emulate part of the Zeeman line broadening in intensity by an increased macroturbulence. The HD-FR exhibited a negligible macroturbulence, with $v_{\mathrm{mac}}<10 \mathrm{~m} \mathrm{~s}^{-1}$ on $90 \%$ of the pixels and an average $v_{\text {mac }}$ of $0.4 \mathrm{~km} \mathrm{~s}^{-1}$ on the remaining $10 \%$ of the FOV. The few pixels with increased $v_{\text {mac }}$ in the HDFR were almost exclusively located inside intergranular lanes.

The macroturbulent velocity introduced into the synthetic spectra by the effect of spatial degradation alone (left middle panel of Fig. 13) has average values increasing from 0.6 to $0.9 \mathrm{~km} \mathrm{~s}^{-1}$ with decreasing spatial resolution (Table 5). The value of $v_{\text {mac }}$ was significant on all pixels in the FOV of the HD-SPAT. The macroturbulent velocity thus becomes significant between the spatial sampling of the HD simulation of $0 .^{\prime} 13$ and the highest spatial resolution in the observations of 0 !' 3 . The spatial structuring of $v_{\text {mac }}$ in the HD-SPAT resembled an inverse granulation pattern, but only remotely. 
Beck et al.: Thermodynamic fluctuations in photospheric convection simulations and observations
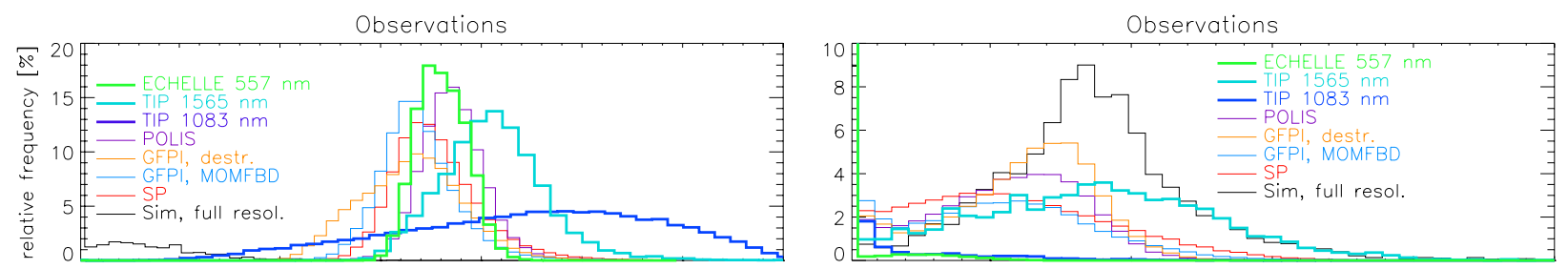

Spatially degraded simulation
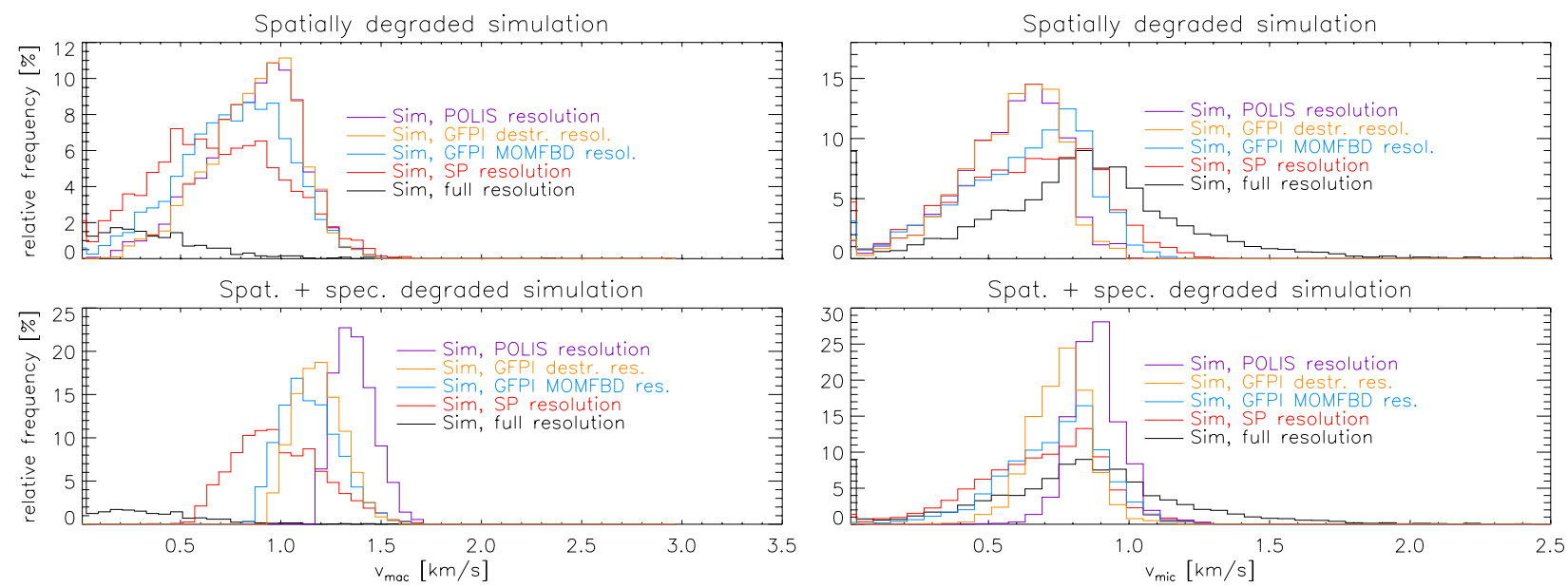

Fig. 13. Histograms of the macroturbulent (left column) and microturbulent velocity (right column) in the inversions. Top: inversions of observations. Middle: the same for the HD-SPAT simulation. Bottom: the same for the HD-SPAT-SPEC. Thin black lines in each panel: HD-FR.

Table 5. Average and $\mathrm{rms}^{1}$ of the macroturbulent and microturbulent velocity in $\mathrm{km} \mathrm{s}^{-1}$, and $\mathrm{rms}$ of the LOS velocity in the inversion.

\begin{tabular}{|c|c|c|c|c|c|c|c|}
\hline \multicolumn{8}{|c|}{ Observations } \\
\hline Data & SP & GFPI, MOMFBD & GFPI, destr. & POLIS & TIP@1083 nm & TIP@1565nm & $557.6 \mathrm{~nm}$ \\
\hline$v_{\mathrm{mac}}$ & $1.81 \pm 0.23$ & $1.72 \pm 0.20$ & $1.68 \pm 0.25$ & $1.88 \pm 0.16$ & $2.27 \pm 0.65$ & $2.11 \pm 0.34$ & $1.83 \pm 0.12$ \\
\hline$v_{\text {mic }}$ & $(0.56 \pm 0.38)^{2}$ & $(0.47 \pm 0.36)^{2}$ & $(0.57 \pm 0.32)^{2}$ & $(0.50 \pm 0.31)^{2}$ & $(0.06 \pm 0.16)^{2}$ & $(0.83 \pm 0.48)^{2}$ & $(0.25 \pm 0.20)^{2}$ \\
\hline$v_{\mathrm{mic}}>10 \mathrm{~m} \mathrm{~s}^{-1}$ & $31 \%$ & $34 \%$ & $52 \%$ & $29 \%$ & $15 \%$ & $42 \%$ & $<1 \%$ \\
\hline $\operatorname{rms}\left(v_{\mathrm{LOS}}\right)$ & 744 & 443 & 362 & 444 & 497 & 500 & 319 \\
\hline \multicolumn{7}{|c|}{ Spatially degraded HD simulation } & $\mathrm{SIM}^{3}$ \\
\hline$v_{\mathrm{mac}}$ & $0.63 \pm 0.39$ & $0.78 \pm 0.30$ & $0.89 \pm 0.23$ & $0.88 \pm 0.24$ & & & $(0.41 \pm 0.32)^{4}$ \\
\hline$v_{\text {mic }}$ & $0.55 \pm 0.34$ & $0.57 \pm 0.31$ & $0.58 \pm 0.22$ & $0.58 \pm 0.22$ & & & $0.76 \pm 0.45$ \\
\hline $\operatorname{rms}\left(v_{\mathrm{LOS}}\right)$ & 686 & 534 & 405 & 416 & & & 1058 \\
\hline \multicolumn{8}{|c|}{ Spatially and spectrally degraded HD simulation } \\
\hline$v_{\text {mac }}$ & $1.03 \pm 0.22$ & $1.18 \pm 0.15$ & $1.22 \pm 0.12$ & $1.40 \pm 0.10$ & & & \\
\hline$v_{\text {mic }}$ & $0.68 \pm 0.27$ & $0.76 \pm 0.19$ & $0.79 \pm 0.11$ & $0.91 \pm 0.10$ & & & \\
\hline $\operatorname{rms}\left(v_{\mathrm{LOS}}\right)$ & 685 & 529 & 400 & 410 & & & \\
\hline
\end{tabular}

Notes. ${ }^{(1)}$ Numbers in parentheses indicate that the average was done only over the fraction of the FOV where the quantity was significant $\left(>10 \mathrm{~m} \mathrm{~s}^{-1}\right)$; ${ }^{(2)}$ only $v_{\text {mic }}>10 \mathrm{~m} \mathrm{~s}^{-1} ;{ }^{(3)} 630 \mathrm{~nm}$ data at full resolution without any degradation; ${ }^{(4)}$ only $v_{\operatorname{mac}}>10 \mathrm{~m} \mathrm{~s}^{-1}, 10 \%$ of the FOV.

The inversion of the HD-SPAT-SPEC spectra (left bottom panel of Fig. 13) yielded an average $v_{\text {mac }}$ lower by about $0.4-0.8 \mathrm{~km} \mathrm{~s}^{-1}$ than for the observations. The final values from the inversion of the HD simulation agree well with the quadratic addition of the two contributions from the spatial and spectral degradation, indicating that the macroturbulent velocity retrieved by the inversion is a rather "robust" quantity that reliably captures the different broadening contributions. As an example, for the HD simulation degraded to SP resolution one has $\left\langle v_{\mathrm{mac}}\right\rangle=1.03 \mathrm{~km} \mathrm{~s}^{-1} \sim \sqrt{0.83^{2}+0.63^{2}}=1.04 \mathrm{~km} \mathrm{~s}^{-1}$ (Tables 3 and 5), which nevertheless falls short of the value in the observations $\left(1.81 \mathrm{~km} \mathrm{~s}^{-1}\right)$.

Microturbulent velocity The microturbulent velocity $v_{\text {mic }}$ in the HD simulation and the observations behaved roughly opposite to $v_{\text {mac }}$. The value of $v_{\text {mic }}$ stayed below $10 \mathrm{~m} \mathrm{~s}^{-1}$ on a large fraction of $50-85 \%$ of the FOV for all observations (Table 5, top part), contrary to $v_{\mathrm{mac}}$. It was instead significant on every pixel in the inversions of the synthetic HD-FR. The average microturbulent velocity for simulations with any type of degradation exceeded the value in the observation - again contrary to the behaviour of macroturbulent velocity. The spatial maps of $v_{\text {mic }}$ for the HD-FR spectra exhibited a granular pattern, whereas those of the observations resembled $v_{\text {mac }}$ of the HD-FR, i.e. for the observational data $v_{\text {mic }}$ was only significant in a few points in intergranular lanes. The HD-SPAT yielded a nearly identical average $v_{\text {mic }}$ of about $0.57 \mathrm{~km} \mathrm{~s}^{-1}$ regardless of the amount of spatial degradation. After the additional spectral degradation, $v_{\text {mic }}$ increases with decreasing spatial resolution (Table 5, bottom part) as for $v_{\text {mac }}$.

Despite the effort in matching synthetic and observed spectra spatially and spectrally, the inversion with SIR retrieves a quite different behaviour with respect to $v_{\text {mac }}$ and $v_{\text {mic }}$. The reason most likely is the usage of the Unsöld approximation by LILIA in the 

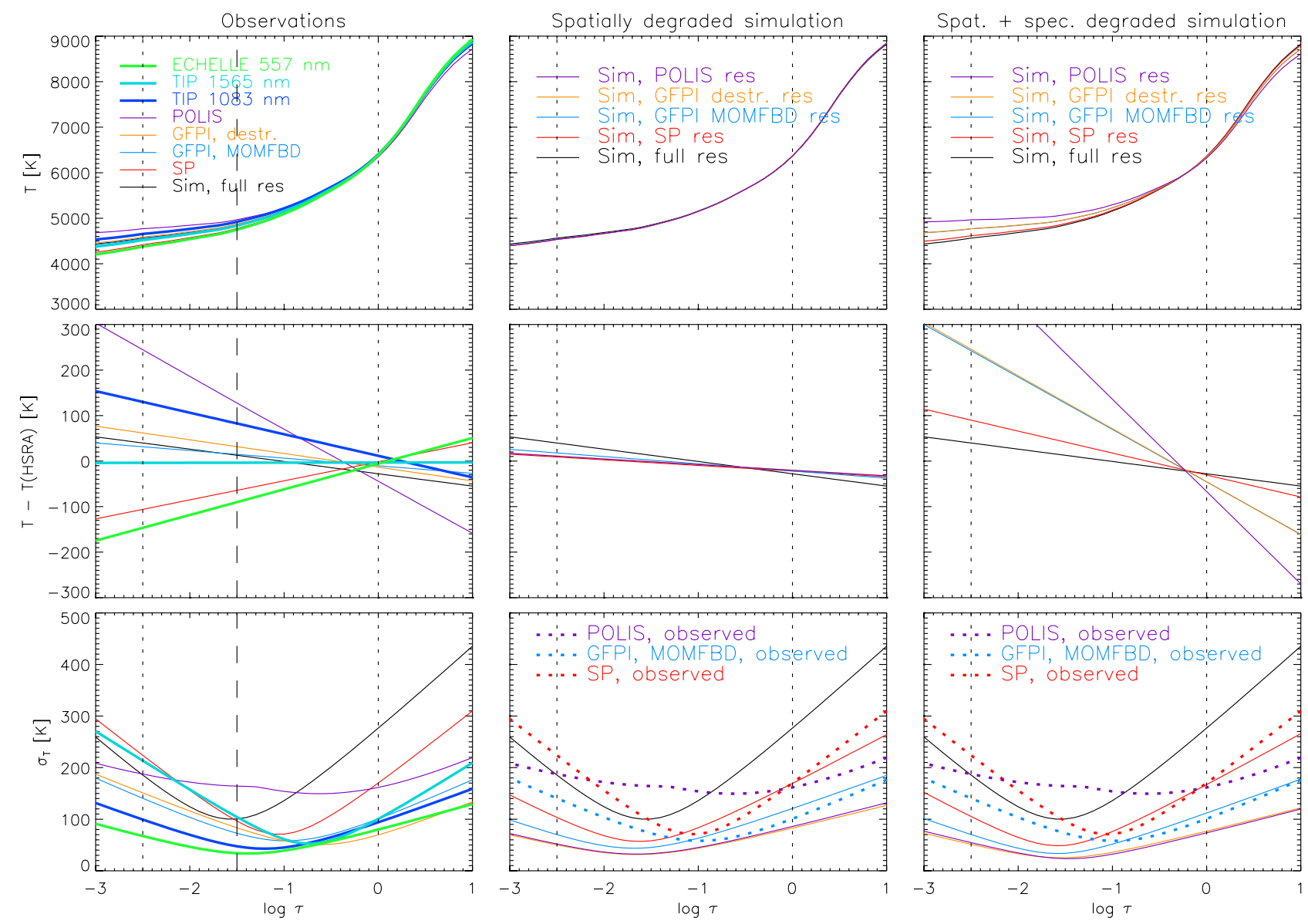

Fig. 14. Temperature stratifications (top row), difference to the HSRA model (middle row), and rms fluctuations of temperature (bottom row) in the inversions. Left to right: observations, HD-SPAT and HD-SPAT-SPEC. The dotted vertical lines denote the approximate formation height range of the $630 \mathrm{~nm}$ lines, the dashed vertical line the upper end of the range for near-IR lines. Other labels are identical to Fig. 13.

synthesis, whereas SIR used the ABO formalism in the inversion of the observed or synthetic spectra. In any case, this should not have had a significant influence on the temperature fluctuations retrieved by the inversion.

Temperature The last indicators of thermodynamics provided by the inversion are the temperature stratifications $T$, and - more relevant for the current study - the rms fluctuations $\sigma_{T}(\tau)$ of the temperature at a given optical depth. Figure 14 shows the average temperature stratifications (top), their deviation from the HSRA model (middle), and their rms fluctuations (bottom) vs. optical depth for the observations (left column), the HDSPAT (middle column), and the HD-SPAT-SPEC (right column).

The average temperature stratifications retrieved from the inversion of the observations differ somewhat (top left panel), but overlap between them or with the HSRA model within $\lesssim \pm 100 \mathrm{~K}$ in the range of $\log \tau=0$ to -1 (left middle panel of Fig. 14). For $\log \tau<-1$, they show a considerably larger scatter between the different instruments and increasing temperature deviations. The slope (from lower to higher optical depth) of the deviation from the HSRA changes from being positive for the SP data to slightly negative for the GFPI data, and finally becomes strongly negative for the POLIS data. The temperature stratification retrieved by inverting the $630 \mathrm{~nm}$ spectra from the HDFR would correspond roughly to the average of all other stratifications derived from the observations. The deviation (from the HSRA model) of the average temperature stratification retrieved from inverting the HD-FR shows a slightly positive slope, with differences of $\lesssim \pm 50 \mathrm{~K}$.

We note that the trend in the slope of the deviation from HSRA (SP $<$ GFPI $<$ POLIS) in the case of the inversion of the observational data does not follow the spatial resolution trend of the latter (GFPI $<$ SP $<$ POLIS). A different suspect instead clearly comes to mind for which the trend is the same as for the slopes: namely, the stray-light offset inside the respective spectrometers (cf. Table 3 ). Whereas $v_{\text {mic }}$ and $v_{\text {mac }}$ can account for the spectral broadening in each instrument, the constant intensity offset $\beta$ was not considered in the inversion. The hypothesis that the slope is related to the spectral stray-light offset is further confirmed when considering the inversions of the HD simulation degraded to GFPI resolution, where we have two cases with different spatial but identical spectral resolution. The deviations from HSRA after degrading the HD simulation only spatially to the spatial resolution of the MOMFBD or to the destretched GFPI spectra stay small and both slopes are almost identical (central panel, lines are overlapping) even if the spatial resolution is clearly different (Table 1 and Fig. 7). After the additional application of the spectral degradation to the spatially degraded HD simulation spectra, the slope of the deviation from HSRA increases significantly for the synthetic data at GFPI resolution (middle right panel), lying between that of the HD simulation at SP or POLIS resolution, but with again little difference between the two different spatial resolutions of the GFPI data (orange and blue line overlapping again). This indicates that, contrary to the spatial resolution, the spectral resolution has a strong effect on 
the deviation from HSRA, likely due to stray-light effects unaccounted for in the inversions.

The rms fluctuations of $T$ are shown in the bottom row of Fig. 14. For the observations (bottom left panel), the values range from 100 to $200 \mathrm{~K}$ at $\log \tau=0$, pass through a minimum of below $100 \mathrm{~K} \mathrm{rms}$ between $\log \tau \sim-0.7$ and -1.2 , and tend to increase to up to $\sim 300 \mathrm{~K}$ at $\log \tau \lesssim-2.5$. This agrees with the results obtained by Puschmann et al. (2003, 2005), who found a minimum temperature contrast between granules and IGLs at $\log \tau=-1$. The HD-FR yields about $100 \mathrm{~K}$ larger fluctuations in the lower atmosphere up to $\log \tau=-1$, but lower rms values than for instance the SP data above that height. The shape of the rms curve for the HD simulation is similar to that of the inversion of, e.g., the SP data, besides an offset in log $\tau$ of about 0.75 (compare the location of minimal $\mathrm{rms}$ in both curves). This offset could be caused by different values of gas (or electron) densities because for instance in the inversion with SIR the electron density at $\log \tau=+1.4$ was fixed to the value in the HSRA as a boundary condition. After the spatial degradation of the HD simulation, the rms temperature fluctuations retrieved from the inversion with SIR match the observations in the lower photosphere from $\log \tau=0$ to -1 well (compare the red/blue solid and red/blue-dotted curves in the bottom middle panel of Fig. 14), whereas they are smaller than the corresponding observed rms values for higher layers. The subsequent spectral degradation has no additional visible effect on the rms temperature fluctuations (bottom right panel), while it significantly changes the average temperature stratification, hence producing the difference to the HSRA (middle right panel).

The scatter of the average temperature stratifications retrieved from the HD-SPAT-SPEC becomes comparable to that between the different observations (top left and top right panels). The deviations between the average temperature stratification from the inversion of the HD-SPAT-SPEC and the HSRA (middle right panel) follow the same systematic trend as in the case of the observations (middle left panel), i.e. a decrease in the slope from SP to POLIS, but in this case starting already from a negative slope for the SP data. That the HD-SPAT-SPEC show the same trend as the observations - while the HD-SPAT (central panel) shows a basically zero slope - again confirms that the spectral degradation, and there mainly the stray-light offset, are responsible for the variation in the derived temperature stratifications. The stray-light offset changes mainly the line depth, and therefore compresses or expands the attributed optical depth scale in the inversion.

The deviations from the HSRA (or any other semi-empirical reference model) would be a concern for an abundance determination, but in the present study we are only interested in the behaviour of observations and simulations relative to each other when using the same analysis approach for both. In particular, we focus on the rms fluctuations rather than on the average values. In that respect, the rms temperature fluctuations as derived from the degraded HD simulation do not exceed the observed properties. Rather, they match them fairly well at layers of formation of photospheric spectral lines commonly used for abundance derivations, and actually are smaller than the observed ones at high layers.

\subsection{Comparison of temperature fluctuations to other studies}

The temperature fluctuations retrieved by the inversion of the degraded HD spectra do not exceed the observed values. To investigate whether this could be caused by the amplitude of fluctuations in our simulations being too small to start with, we
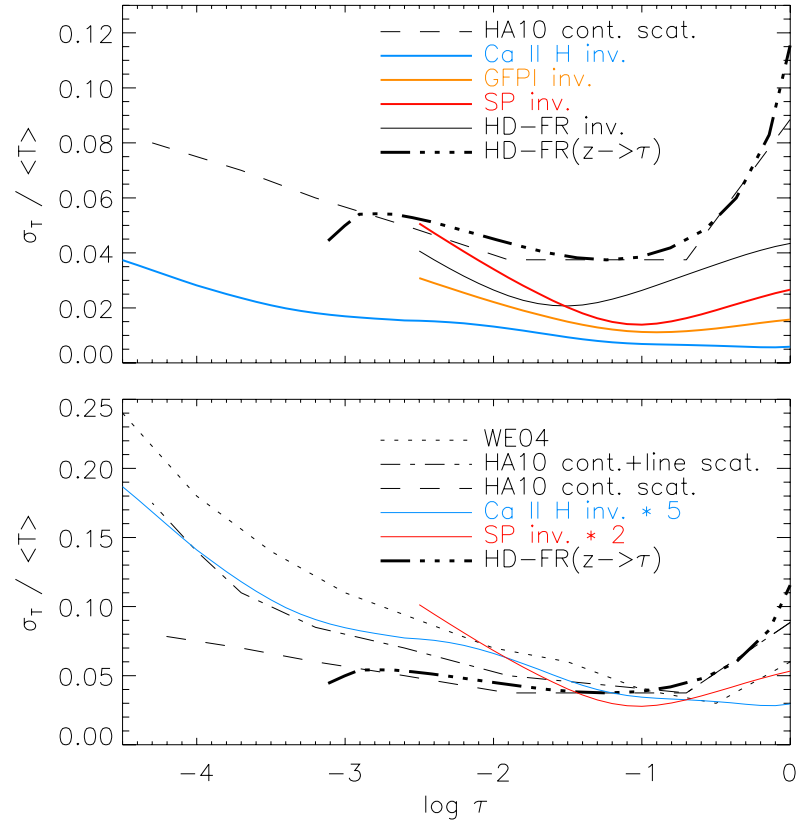

Fig. 15. Relative temperature fluctuations vs. optical depth. Top panel: inversion results of SP (red line), GFPI (orange line) and Ca II H data (blue line), and of the HD-FR simulation spectra (black-solid line). Overplotted are the relative temperature fluctuations in the HD-FR simulation box (black dashed-triple-dotted line) and the curve given in Hayek et al. (2010, HA10) for a simulation with continuum scattering (black-dashed line). Bottom panel: inversion results of SP/Ca II H multiplied by two/five (red/blue lines), and the values from the simulations of Wedemeyer et al. (2004, WE04; black-dotted line), of HA10 with continuum scattering (black-dashed line) and of HA10 with continuum and line scattering (black-dash-dotted line).

compared the temperature fluctuations in our data (simulations and observations), with the values provided for other simulations (Fig. 15). All curves obtained from an inversion of $630 \mathrm{~nm}$ spectra were clipped at $\log \tau=-2.5$ because the formation height of the lines does not cover higher layers. To extend the height range covered from the observational side, we included the LTE inversion results of Ca II H spectra from Beck et al. (2013a,b).

We compare our values with those given in Wedemeyer et al. (2004, WE04; their Fig. 9) and Hayek et al. (2010, HA10; their Fig. 10) because they correspond to different treatments of the radiative transfer in the simulations, i.e. grey atmosphere in LTE (WE04) and LTE with additional continuum and line scattering (HA10). Because these simulations were not spatially degraded, we overplotted the results for the inversion of the SP (Ca II H) data multiplied by two (five) in the lower panel of Fig. 15. These factors roughly correspond to the ratio of continuum contrast between observations and simulations, e.g. for the SP (Ca II H) one has an observed rms $I_{\mathrm{c}}=7.2 \%(3.5 \%)$, whereas simulations predict about $15 \%$ (>20\%; Hirzberger et al. 2010). We used the tabulated $z-\tau$ relation in the HSRA model to convert curves vs. geometrical height $(\operatorname{HA} 10, \operatorname{HD}-\mathrm{FR}(z \rightarrow \tau))$ to an optical depth scale. This approach can slightly compress or stretch the curves, when the true density stratification differs from the one of the HSRA.

When comparing the curves of the temperature rms derived from observations and simulations, the best match is achieved between the one derived from $\mathrm{Ca}$ II $\mathrm{H}$, after scaling it up for the spatial resolution, and the results of HA10 including continuum and line scattering (lower panel of Fig. 15). The rms fluctuations in WE04 exceed all others above $\log \tau=-1$. The temperature fluctuations in the Stagger code simulations that we used 
closely match the HA10 case with only continuum scattering included. The rms fluctuations of all simulations are comparable up to about $\log \tau=-2$ with relative fluctuations between 5 and $7.5 \%$ at this optical depth, but increasingly deviate in higher layers with differences of up to $15 \%$ at $\log \tau=-4$ (三up to $600 \mathrm{~K}$ difference in rms). From the comparison, we would exclude that the Stagger simulations that we analysed here are somehow biased towards an extraordinary low or high level of fluctuations in the photosphere up to $\log \tau=-2$, which is about the maximal height range where the spectral lines used here are fully reliable.

\section{Summary and discussion}

Numerical (M)HD simulations are increasingly used in the analysis or interpretation of observational data of the Sun (e.g. Khomenko et al. 2005; Shelyag et al. 2007; Carroll \& Kopf 2008) and in particular also for the derivation of abundances (Asplund et al. 2004; Caffau et al. 2008; Pereira et al. 2009a; Fabbian et al. 2010, 2012). They are capable of predicting phenomena such as small-scale vortices (e.g. Stein \& Nordlund 1998; Moll et al. 2011; Kitiashvili et al. 2011) that have been confirmed by observations only later on (Bonet et al. 2008; Steiner et al. 2010; Steiner \& Rezaei 2012). The claim that a simulation is "realistic" is, however, often made solely based on the physics included in the numerical calculation, and not necessarily because the simulations were proven to correspond to the "real" Sun as far as can be determined from observations. Several different tests of the realism of simulations were performed in the past, but mainly concentrating on a few selected (average) parameters such as the continuum contrast (Wedemeyer-Böhm \& Rouppe van der Voort 2009; Hirzberger et al. 2010), its CLV (Koesterke et al. 2008), average profiles (Asplund et al. 2000; Stein \& Nordlund 2000; Allende Prieto et al. 2004) and their CLV (Asplund et al. 2004), or average temperature stratifications (e.g. Pereira et al. 2013). Only recently, spatially resolved properties of individual line profiles were also used for comparisons of simulations and observations (e.g. Pereira et al. 2009b,c). Additional cross-checks have recently been done between different simulation codes (e.g. Beeck et al. 2012), rather than between simulations and observations. Some of the plots in Beeck et al. (2012) can, however, be directly compared to those in the present study. We only point out one feature of the temperature rms vs. $\log \tau$ (their Fig. 12), namely the location of minimum rms at about $\log \tau \sim-0.7$, compared to $\log \tau \sim-1$ in our inversions of the observed spectra, with a similar result also in the inversions of Puschmann et al. (2005).

All types of comparisons between simulations and observations usually share the same problem: the spatial resolution of the observations is not as good as that of the simulations, and thus it can only be shown that the simulations can be degraded sufficiently to match the observations, without the possibility to investigate if the original simulation results match the "real" Sun (cf. also Danilovic et al. 2008). Our present study demonstrated that to do this final step would require observational data with about three to four times better spatial resolution than currently available. Such data will be available soon with the upcoming telescope projects of the 1.5-m class such as the GREGOR solar telescope (e.g. Schmidt et al. 2012a,b) with the GREGOR FabryPerót Interferometer (e.g. Puschmann et al. 2012a,b,c) and the New Solar Telescope (Denker et al. 2006; Cao et al. 2010), or in the future with next generation solar telescopes of the 4-m class such as ATST (Wagner et al. 2008; Rimmele et al. 2010) or EST (Collados et al. 2010).
Our investigation shows that using 3D convection simulations as reference allows one to derive an estimate of the spatial point spread function of instruments and telescopes that can be used for an accurate stray-light correction and/or for a spatial deconvolution (BE11, Löfdahl \& Scharmer 2012). The good match to a directly measured instrumental PSF (Fig. 5) implies that the use of the simulations for this purpose is justified. The importance of knowing the instrumental PSF should not be underestimated. The inversion of the HD-SPAT shows that the spatial smearing introduces a significant broadening of spectral lines already at a resolution of 0.3 . Taking the PSF explicitly into account in the analysis of data will thus yield more accurate solar properties in an inversion of spectra not only for thermodynamic parameters, but also for magnetic field properties. The PSF is commonly taken into account to correct for unpolarized stray light only, but naturally applies as well to polarized stray light, where it has a more profound impact on retrieved solar parameters, e.g. for all spectral lines in the weak-field limit where the polarization amplitude is proportional to the total magnetic flux, or wherever spatial resolution is critical because of solar finestructure such as in the penumbrae of sunspots (van Noort 2012).

The comparison of the HD-SPAT-SPEC data to the observations yielded a fair to very good match for most line parameters. The HD-FR differs usually only by an increased amplitude of the quantities, with the exception of the average bisector position (Fig. 11). For this parameter, we find that its average values at different line depression levels trace a curve that for the HDFR has a roughly reverse shape compared to the one for the spatially degraded HD simulation and the ones corresponding to the different observational data. This change thus has to happen on a spatial scale between $0 . ' 13$ and $0 . ' 3$, close to the photon mean free-path length in the solar photosphere. Given the corresponding thermalization length, one does not expect significant spatial temperature variations in the photosphere on spatial scales much smaller than $0.13(\sim 100 \mathrm{~km})$, but this argument does not hold in the same way for spatial velocity variations. The presence of Doppler shifts also enlarges the thermalization length (Skartlien 2000). The characteristic properties of the average bisector come from an intensity-weighted spatial average of the corresponding velocities. The difference in shape between the bisector derived from the HD-FR and those derived from any observed spectra then presumably is caused by both mass flows and spatial temperature variations that remain unresolved in the observations, while the spatial temperature variations should be partially resolved or close to being resolved in our best observations.

The bisector flips to the observed shape with any large-scale averaging. Our finding therefore should have no impact on abundance determinations from averaged spectra. It, however, raises the warning that, since the characteristic properties of spatially averaged spectral line profiles derived from observations do not necessarily reflect those of spatially resolved profiles, achieving a match with the former is not fully sufficient to prove the realism of simulations. Many line parameters such as bisectors depend on the exact line shape caused by gradients of thermodynamic parameters. A spatial averaging can destroy the (non-linear) dependence of the profile shape on the thermodynamic parameters.

We point one peculiarity of the often used atlas spectra such as the FTS: the smearing of these data due to spatial averaging and the corresponding amount of macroturbulent broadening are practically unknown. Spatial smearing introduces a significant line broadening of $v_{\mathrm{mac}} \sim 1 \mathrm{~km} \mathrm{~s}^{-1}$ at a resolution of 0.3 , as the inversion of the HD-SPAT showed. In the case of spatially resolved observations, one can estimate the characteristic macroturbulent line broadening of the data in the resolved 
and averaged spectra. For the atlas spectra, this estimate cannot be done post-facto. The commonly adopted implicit assumption that the broadening by macroturbulent velocities levels off to a constant value at some degree of averaging should be investigated in more detail.

The simulation box covered a height range up to more than $400 \mathrm{~km}$ above optical depth unity in the solar atmosphere, and the spectral lines used in this study - besides the Si I line at $1082.7 \mathrm{~nm}$ that is not fully reproduced - form in the low to mid photosphere. The variation of quantities with height, like for instance a monotonic decrease in rms velocities, indicates that the values retrieved from the spectral lines still belong to an atmospheric regime whose dynamics are governed by the convective energy transport. Any possible flaw of the numerical simulations because of the approximations used (namely, LTE and opacity binning treatment) for the radiative energy transfer and losses is thus less relevant in these layers because the directed mass motions control the energy balance. A similar study covering also (low) chromospheric layers, e.g. up to a height of $1 \mathrm{Mm}$, should, however, not be too difficult to achieve in the near future (cf. Wedemeyer et al. 2004; Leenaarts et al. 2007, 2009; Wedemeyer-Böhm \& Carlsson 2011).

The inversion of the HD-SPAT yielded a small $(\$ \pm 50 \mathrm{~K})$ spread in the resulting average temperature stratifications even if the spatial resolution varied between 0.3 and about $1^{\prime \prime}$ (central panel of Fig. 14). The spectral degradation, on the other hand, led to a strong variation in the retrieved temperature stratifications, with a clear scaling by the constant wavelength-independent stray-light offset $\beta$ inside the spectrometer. This implies that for an accurate derivation of temperatures from observed spectra the stray-light offset has to be corrected before an inversion. On the positive side, the stray-light offset $\beta$ inside the SP spectrograph was found to be below 3\% (see also Lites et al. 2013), which makes these data and data of similar spectral quality well suited for a derivation of temperature stratifications in the solar photosphere (e.g. Socas-Navarro 2011).

The size of the statistical samples of simulations and observations in our case are not identical. The FOV of the simulations of $(6 \mathrm{Mm})^{2}$ covers only a small fraction of that of the observational data used. The close match between characteristic quantities indicates that any statistical effect should, however, not have a significant impact. The line properties change drastically with the spatial resolution, but in the same way in degraded simulations and observations. An inclusion of more simulation snapshots therefore is likely to confirm the close match between the statistics of line parameters from observations and degraded simulations. As a test, we compared the line profile resulting from averaging over one or over 21 snapshots covering the whole HD simulation series. The corresponding average profiles differed only slightly, maintaining similar differences towards FTS or the observed spectra as those seen in Fig. 8.

\section{Conclusions}

We find no indications that the thermodynamic fluctuations in a state-of-the-art numerical hydrodynamical simulation exceed those in observations of the solar photosphere when the simulation is spatially degraded to a resolution $\geq 0$ ' 3 . For a final proof that the initial properties of the simulation at full resolution do not exceed solar surface properties, observations that resolve the photon mean free-path length $\left(<00^{\prime} 1\right)$ are required. Solar telescopes with free apertures of about $1.5 \mathrm{~m}$ and next-generation solar telescopes of the 4-m class are designed to achieve this objective.

Acknowledgements. The VTT is operated by the Kiepenheuer-Institut für Sonnenphysik (KIS) at the Spanish Observatorio del Teide of the Instituto de Astrofísica de Canarias (IAC). The POLIS instrument has been a joint development of the High Altitude Observatory (Boulder, USA) and the KIS. Hinode is a Japanese mission developed and launched by ISAS/JAXA, collaborating with NAOJ as a domestic partner, NASA and STFC (UK) as international partners. Scientific operation of the Hinode mission is conducted by the Hinode science team organized at ISAS/JAXA. This team mainly consists of scientists from institutes in the partner countries. Support for the postlaunch operation is provided by JAXA and NAOJ (Japan), STFC (UK), NASA, ESA, and NSC (Norway). C.B. acknowledges partial support by the Spanish Ministerio de Ciencia e Innovación through project AYA 2010-18029. R.R. acknowledges financial support by the DFG grant RE 3281/1-1. D.F. gratefully acknowledges financial support by the European Commission through the SOLAIRE Network (MTRN-CT-2006-035484) and by the Programa de Acceso a Grandes Instalaciones Científicas financed by the Spanish Ministerio de Ciencia e Innovación. The latter is also thanked by D.F. and F.M.-I. for providing funds through the related projects AYA2007-66502, CSD2007-00050, AYA2007-63881 and AYA2011-24808. The simulations were possible thanks to time awarded on the MareNostrum (BSC/CNS, Spain), the Danish Centre for Scientific Computing (DCSC-KU, Denmark), La Palma (IAC/RES, Spain) and the HRLS/DEISA (Germany) supercomputer installations. We thank A. Asensio Ramos and A. Lagg for their helpful comments on the present article.

\section{References}

Afram, N., Unruh, Y. C., Solanki, S. K., et al. 2011, A\&A, 526, A120

Allende Prieto, C., Barklem, P. S., Asplund, M., \& Ruiz Cobo, B. 2001, ApJ, 558,830

Allende Prieto, C., Asplund, M., \& Fabiani Bendicho, P. 2004, A\&A, 423, 1109 Asplund, M. 2005, ARA\&A, 43, 481

Asplund, M., Nordlund, Å., Trampedach, R., Allende Prieto, C., \& Stein, R. F. 2000, A\&A, 359, 729

Asplund, M., Carlsson, M., \& Botnen, A. V. 2003, A\&A, 399, L31

Asplund, M., Grevesse, N., Sauval, A. J., Allende Prieto, C., \& Kiselman, D. 2004, A\&A, 417, 751

Asplund, M., Grevesse, N., \& Sauval, A. J. 2005, in Cosmic Abundances as Records of Stellar Evolution and Nucleosynthesis, eds. T. G. Barnes III, \& F. N. Bash, ASP Conf. Ser., 336, 25

Asplund, M., Grevesse, N., Sauval, A. J., \& Scott, P. 2009, ARA\&A, 47, 481

Bahcall, J. N., Basu, S., \& Serenelli, A. M. 2005, ApJ, 631, 1281

Bard, S., \& Carlsson, M. 2008, ApJ, 682, 1376

Barklem, P. S., Piskunov, N., \& O’Mara, B. J. 2000, A\&AS, 142, 467

Beck, C. 2011, A\&A, 525, A133

Beck, C., \& Rezaei, R. 2009, A\&A, 502, 969

Beck, C., Schlichenmaier, R., Collados, M., Bellot Rubio, L., \& Kentischer, T. 2005a, A\&A, 443, 1047

Beck, C., Schmidt, W., Kentischer, T. \& Elmore, D. 2005b, A\&A, 437, 1159

Beck, C., Khomenko, E., Rezaei, R., \& Collados, M. 2009, A\&A, 507, 453

Beck, C., Rezaei, R., \& Fabbian, D. 2011, A\&A, 535, A129 (BE11)

Beck, C., Rezaei, R., \& Puschmann, K. G. 2013a, A\&A, 549, A24

Beck, C., Rezaei, R., \& Puschmann, K. G. 2013b, A\&A, 553, A73

Beeck, B., Collet, R., Steffen, M., et al. 2012, A\&A, 539, A121

Bello González, N., \& Kneer, F. 2008, A\&A, 480, 265

Bethge, C., Peter, H., Kentischer, T. J., et al. 2011, A\&A, 534, A105

Bethge, C., Beck, C., Peter, H., \& Lagg, A. 2012, A\&A, 537, A130

Bonet, J. A., Márquez, I., Sánchez Almeida, J., Cabello, I., \& Domingo, V. 2008, ApJ, 687, L131

Brandt, P. N., \& Schroeter, E. H. 1982, Sol. Phys., 79, 3

Briand, C., Mattig, W., Ceppatelli, G., \& Mainella, G. 2006, Sol. Phys., 234, 187

Cabrera Solana, D., Bellot Rubio, L. R., \& del Toro Iniesta, J. C. 2005, A\&A 439,687

Cabrera Solana, D., Bellot Rubio, L. R., Beck, C., \& Del Toro Iniesta, J. C. 2007, A\&A, 475, 1067

Caffau, E., Steffen, M., Ayres, T. R., et al. 2008, A\&A, 488, 1031

Cao, W., Gorceix, N., Coulter, R., Coulter, A., \& Goode, P. R. 2010, in Groundbased and Airborne Telescopes III, eds. L. M. Stepp, G. R., \& H. J. Hall, SPIE Conf. Ser., 7733

Carlsson, M., \& Leenaarts, J. 2012, A\&A, 539, A39

Carroll, T. A., \& Kopf, M. 2008, A\&A, 481, L37

Chapman, G. A. 1977, ApJS, 33, 35

Collados, M., Lagg, A., Díaz Garcí A, J. J., et al. 2007, in The Physics of Chromospheric Plasmas, eds. P. Heinzel, I. Dorotovič, \& R. J. Rutten, ASP Conf. Ser., 368, 611 
Collados, M., Bettonvil, F., Cavaller, L., et al. 2010, in Ground-based and Airborne Telescopes III, eds. L. M. Stepp, G. R., \& H. J. Hall, SPIE Conf. Ser., 7733

Collet, R., Hayek, W., Asplund, M., et al. 2011, A\&A, 528, A32

Danilovic, S., Gandorfer, A., Lagg, A., et al. 2008, A\&A, 484, L17

de la Cruz Rodríguez, J., Kiselman, D., \& Carlsson, M. 2011, A\&A, 528, A113

Delbouille, L., Roland, G., \& Neven, L. 1973, Atlas photometrique DU spectre solaire de [lambda] 3000 a [lambda] 10000 (Liège: Université de Liège, Institut d'Astrophysique)

Denker, C., Goode, P. R., Ren, D., et al. 2006, in Ground-based and Airborne Telescopes, ed. L. M. Stepp, SPIE Conf. Ser., 6267

Dravins, D. 1982, ARA\&A, 20, 61

Dravins, D. 2008, A\&A, 492, 199

Fabbian, D., Asplund, M., Barklem, P. S., Carlsson, M., \& Kiselman, D. 2009a, A\&A, 500, 1221

Fabbian, D., Nissen, P. E., Asplund, M., Pettini, M., \& Akerman, C. 2009b, A\&A, 500, 1143

Fabbian, D., Khomenko, E., Moreno-Insertis, F., \& Nordlund, Å. 2010, ApJ, 724,1536

Fabbian, D., Moreno-Insertis, F., Khomenko, E., \& Nordlund, Å. 2012, A\&A, 548, A35

Felipe, T., Khomenko, E., Collados, M., \& Beck, C. 2010, ApJ, 722, 131

Fontenla, J. M., Avrett, E., Thuillier, G., \& Harder, J. 2006, ApJ, 639, 441

Gingerich, O., Noyes, R. W., Kalkofen, W., \& Cuny, Y. 1971, Sol. Phys., 18, 347

Gray, D. F. 2010, ApJ, 721, 670

Grevesse, N. 1984, Phys. Scr. T, 8, 49

Hanslmeier, A., Kučera, A., Rybák, J., Neunteufel, B., \& Wöhl, H. 2000, A\&A, 356,308

Hayek, W., Asplund, M., Carlsson, M., et al. 2010, A\&A, 517, A49

Hirzberger, J., \& Wiehr, E. 2005, A\&A, 438, 1059

Hirzberger, J., Feller, A., Riethmüller, T. L., et al. 2010, ApJ, 723, L154

Holweger, H., \& Mueller, E. A. 1974, Sol. Phys., 39, 19

Kalkofen, W. 2012, Sol. Phys., 276, 75

Khomenko, E. V., Martínez González, M. J., Collados, M., et al. 2005, A\&A, 436, L27

Kitiashvili, I. N., Kosovichev, A. G., Mansour, N. N., \& Wray, A. A. 2011, ApJ, 727, L50

Koesterke, L., Allende Prieto, C., \& Lambert, L. 2008, ApJ, 680, 764

Kosugi, T., Matsuzaki, K., Sakao, T., et al. 2007, Sol. Phys., 243, 3

Kritsuk, A. G., Nordlund, A., Collins, D., et al. 2011, ApJ, 737, 13

Kurucz, R. L., Furenlid, I., Brault, J., \& Testerman, L. 1984, Solar flux atlas from 296 to $1300 \mathrm{~nm}$ (National Solar Observatory, Sunspot, NM)

Lagg, A., Solanki, S. K., Riethmüller, T. L., et al. 2010, ApJ, 723, L164

Leenaarts, J., Carlsson, M., Hansteen, V., \& Rutten, R. J. 2007, A\&A, 473, 625

Leenaarts, J., Carlsson, M., Hansteen, V., \& Rouppe van der Voort, L. 2009, ApJ, 694, L128

Leenaarts, J., Carlsson, M., \& Rouppe van der Voort, L. 2012, ApJ, 749, 136

Lites, B. W., Akin, D. L., Card, G., et al. 2013, Sol. Phys., 283, 579

Löfdahl, M. G., \& Scharmer, G. B. 2012, A\&A, 537, A80

Marquez, I., Bonet, J. A., Vazquez, M., \& Woehl, H. 1996, A\&A, 305, 316

Martínez Pillet, V., Collados, M., Sánchez Almeida, J., et al. 1999, in High Resolution Solar Physics: Theory, Observations, and Techniques, ASP Conf. Ser., 183, 264

Martínez González, M. J., Collados, M., \& Ruiz Cobo, B. 2006, A\&A, 456, 1159

Martínez González, M. J., Collados, M., Ruiz Cobo, B., \& Beck, C. 2008, A\&A, 477,953

Martínez González, M. J., Bellot Rubio, L. R., Solanki, S. K., et al. 2012, ApJ, $758, \mathrm{~L} 40$

Mattig, W. 1971, Sol. Phys., 18, 434

Mattig, W. 1983, Sol. Phys., 87, 187

Meléndez, J., \& Asplund, M. 2008, A\&A, 490, 817

Mikurda, K., Tritschler, A., \& Schmidt, W. 2006, A\&A, 454, 359

Moll, R., Cameron, R. H., \& Schüssler, M. 2011, A\&A, 533, A126

Neckel, H. 1999, Sol. Phys., 184, 421

Nordlund, A. 1982, A\&A, 107, 1

Nordlund, Å., \& Stein, R. F. 1990, Comp. Phys. Commun., 59, 119

Orozco Suárez, D., Bellot Rubio, L. R., del Toro Iniesta, J. C., et al. 2007, ApJ, 670, L61

Pereira, T. M. D., Asplund, M., \& Kiselman, D. 2009a, A\&A, 508, 1403

Pereira, T. M. D., Asplund, M., \& Kiselman, D. 2009b, Mem. S.A. It., 75, 282

Pereira, T. M. D., Kiselman, D., \& Asplund, M. 2009c, A\&A, 507, 417

Pereira, T. M. D., Asplund, M., Collet, R., et al. 2013, A\&A, 554, A118

Puschmann, K. G., \& Beck, C. 2011, A\&A, 533, A21 (PuB11)
Puschmann, K. G., \& Sailer, M. 2006, A\&A, 454, 1011

Puschmann, K., Hanslmeier, A., \& Solanki, S. K. 1999, in Motions in the Solar Atmosphere, eds. A. Hanslmeier, \& M. Messerotti, Astrophysics and Space Science Library, 239, 227

Puschmann, K., Vázquez, M., Bonet, J. A., Ruiz Cobo, B., \& Hanslmeier, A. 2003, A\&A, 408, 363

Puschmann, K. G., Ruiz Cobo, B., Vázquez, M., Bonet, J. A., \& Hanslmeier, A. 2005, A\&A, 441, 1157

Puschmann, K. G., Kneer, F., Seelemann, T., \& Wittmann, A. D. 2006, A\&A, 451,1151

Puschmann, K. G., Kneer, F., \& Domínguez Cerdeña, I. 2007, in Modern solar facilities - advanced solar science, eds. F. Kneer, K. G. Puschmann, \& A. D. Wittmann (Universitätsverlag Göttingen), 151

Puschmann, K. G., Ruiz Cobo, B., \& Martínez Pillet, V. 2010, ApJ, 720, 1417

Puschmann, K. G., Balthasar, H., Bauer, S.-M., et al. 2012a, in The Second ATST-EAST Meeting: Magnetic Fields from the Photosphere to the Corona, eds. T. R. Rimmele, A. Tritschler, F. Wöger, et al., ASP Conf. Ser., 463, 423

Puschmann, K. G., Balthasar, H., Beck, C., et al. 2012b, in Ground-based and Airborne Instrumentation for Astronomy IV, SPIE Conf. Ser., 8446, 79

Puschmann, K. G., Denker, C., Kneer, F., et al. 2012c, Astron. Nachr., 333, 880

Rammacher, W., \& Cuntz, M. 1991, A\&A, 250, 212

Rimmele, T. R., Wagner, J., Keil, S., et al. 2010, in Ground-based and Airborne Telescopes III, eds. L. M. Stepp, G. R., \& H. J. Hall, SPIE Conf. Ser., 7733

Ruiz Cobo, B., \& del Toro Iniesta, J. C. 1992, ApJ, 398, 375

Rutten, R. J. 2003, Radiative Transfer in Stellar Atmospheres, Lect. Notes Utrecht University

Sánchez Almeida, J. 2005, ApJ, 622, 1292

Schmidt, W., von der Lühe, O., Volkmer, R., et al. 2012a, Astron. Nachr., 333, 796

Schmidt, W., von der Lühe, O., Volkmer, R., et al. 2012b, in The Second ATSTEAST Meeting: Magnetic Fields from the Photosphere to the Corona, eds. T. R. Rimmele, A. Tritschler, F. Wöger, et al., ASP Conf. Ser., 463, 365

Schröter, E. H., Soltau, D., \& Wiehr, E. 1985, Vist. Astron., 28, 519

Shchukina, N., \& Trujillo Bueno, J. 2001, ApJ, 550, 970

Shchukina, N., Sukhorukov, A., \& Trujillo Bueno, J. 2012, ApJ, 755, 176

Shelyag, S., Schüssler, M., Solanki, S. K., \& Vögler, A. 2007, A\&A, 469, 731

Shi, J. R., Gehren, T., Butler, K., Mashonkina, L. I., \& Zhao, G. 2008, A\&A, 486,303

Skartlien, R. 2000, ApJ, 536, 465

Socas-Navarro, H. 2001, in Advanced Solar Polarimetry - Theory, Observation, and Instrumentation, ed. M. Sigwarth, ASP Conf. Ser., 236, 487

Socas-Navarro, H. 2011, A\&A, 529, A37

Socas-Navarro, H., Trujillo Bueno, J., \& Ruiz Cobo, B. 2000, ApJ, 530, 977

Stein, R. F., \& Nordlund, A. 1998, ApJ, 499, 914

Stein, R. F., \& Nordlund, A. 2000, Sol. Phys., 192, 91

Stein, R. F., Lagerfjärd, A., Nordlund, A., \& Georgobiani, D. 2011, Sol. Phys., 268, 271

Steiner, O., \& Rezaei, R. 2012, in Fifth Hinode Science Meeting, eds. L. Golub, I. De Moortel, \& T. Shimizu, ASP Conf. Ser., 456, 3

Steiner, O., Grossmann-Doerth, U., Knoelker, M., \& Schuessler, M. 1998, ApJ, 495, 468

Steiner, O., Franz, M., Bello González, N., et al. 2010, ApJ, 723, L180

Stellmacher, G., \& Wiehr, E. 1971, Sol. Phys., 18, 220

Tsuneta, S., Ichimoto, K., Katsukawa, Y., et al. 2008, Sol. Phys., 249, 167

Unsöld, A. 1955, Physik der Sternatmosphären, Mit besonderer Berücksichtigung der Sonne (Berlin: Springer, 2. Auflage)

van Noort, M. 2012, A\&A, 548, A5

van Noort, M., Rouppe van der Voort, L., \& Löfdahl, M. G. 2005, Sol. Phys., 228,191

Vernazza, J. E., Avrett, E. H., \& Loeser, R. 1981, ApJS, 45, 635

Vögler, A. 2004, A\&A, 421, 755

Vögler, A., Bruls, J. H. M. J., \& Schüssler, M. 2004, A\&A, 421, 741

von der Lühe, O., Soltau, D., Berkefeld, T., \& Schelenz, T. 2003, in Innovative Telescopes and Instrumentation for Solar Astrophysics, eds. S. L. Keil, \& S. V. Avakan, SPIE Conf. Ser., 4853, 187

Wagner, J., Rimmele, T. R., Keil, S., et al. 2008, in Ground-based and Airborne Telescopes II, eds. L. M. Stepp, \& R. Gilmozzi, SPIE Conf. Ser., 7012

Wedemeyer, S. 2001, A\&A, 373, 998

Wedemeyer, S., Freytag, B., Steffen, M., Ludwig, H.-G., \& Holweger, H. 2004, A\&A, 414, 1121

Wedemeyer-Böhm, S. 2008, A\&A, 487, 399

Wedemeyer-Böhm, S., \& Carlsson, M. 2011, A\&A, 528, A1

Wedemeyer-Böhm, S., \& Rouppe van der Voort, L. 2009, A\&A, 503, 225 
Beck et al.: Thermodynamic fluctuations in photospheric convection simulations and observations

\section{Appendix A: Line properties at different line depression levels}

\section{A.1. Average values}

Figures A.1 to A.3 show the average values of the line properties at different line depression levels but for the average bisector velocity of the $630.25 \mathrm{~nm}$ line. The average bisector velocity of the $630.15 \mathrm{~nm}$ line is similar to that of $630.25 \mathrm{~nm}$ in Fig. 11. The two near-IR lines (bottom row of Fig. A.1) sample only low layers in the solar atmosphere. For the observations, the curves thus correspond to those from $10 \%$ line depression up to roughly the minimal bisector velocity of the $630 \mathrm{~nm}$ lines at $30 \%$ line depression. The results derived from the HD-FR spectra show nearly straight bisectors for the near-IR lines. For both the $557.6 \mathrm{~nm}$ and $1082.7 \mathrm{~nm}$ line, the line properties close to the continuum ( $<40 \%$ line depression) suffer from the presence of line blends $(557.6 \mathrm{~nm})$ or ill-defined line depression levels $(1082.7 \mathrm{~nm})$ because of the extended line wings. For the latter line, the limited extension of the simulation box is also likely to play a role. The HD-SPAT-SPEC actually gives smoother curves for these lines than the observations themselves. The average intensities at different line depression levels (Fig. A.2) provide little information in themselves because they basically only measure the line depth. The observations and the HD-SPAT-SPEC match well in most cases, with nearly identical values for instance for the $557.6 \mathrm{~nm}$ or $1565.2 \mathrm{~nm}$ lines. The average line widths at different line depression levels (Fig. A.3) show again that the near-IR line only sample the lower range of the atmosphere, with their curves corresponding to those of the $630 \mathrm{~nm}$ lines up to the inflection point at about the $50 \%$ line depression. The observations and the (degraded) HD simulation match well in the general shape of the curves here, whereas the absolute values of the line width slightly differ (cf. the red and red-dashed curves in Fig. A.3). This mismatch in line width was already seen in the average profiles (Fig. 8).
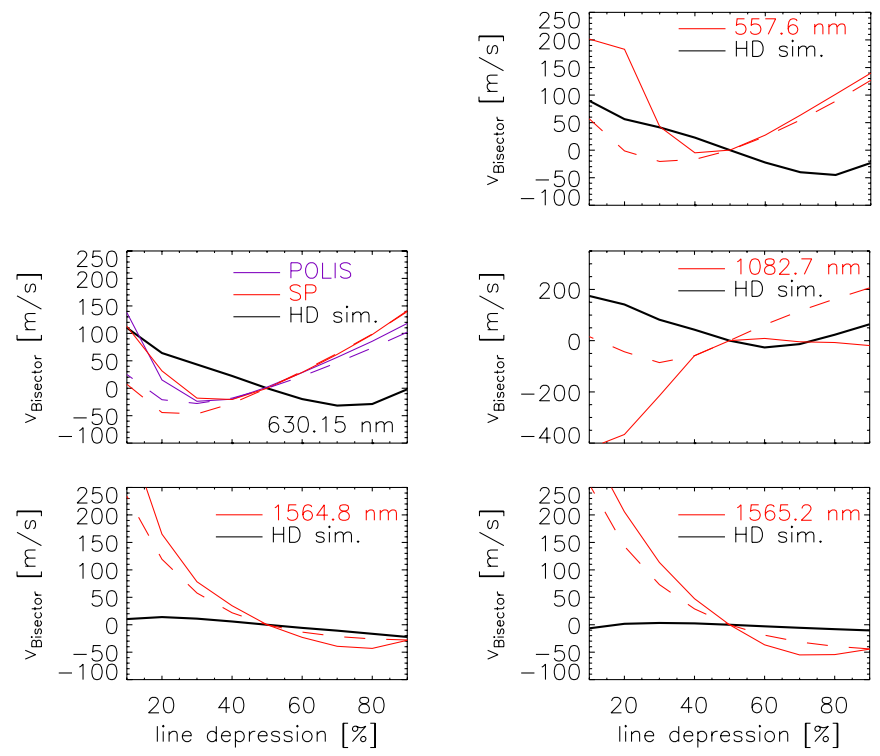

Fig. A.1. Average velocities at different line depression levels for all lines but $630.25 \mathrm{~nm}$ in the observations (solid lines), the HD-SPATSPEC (dashed lines) and the HD-FR (solid black line).
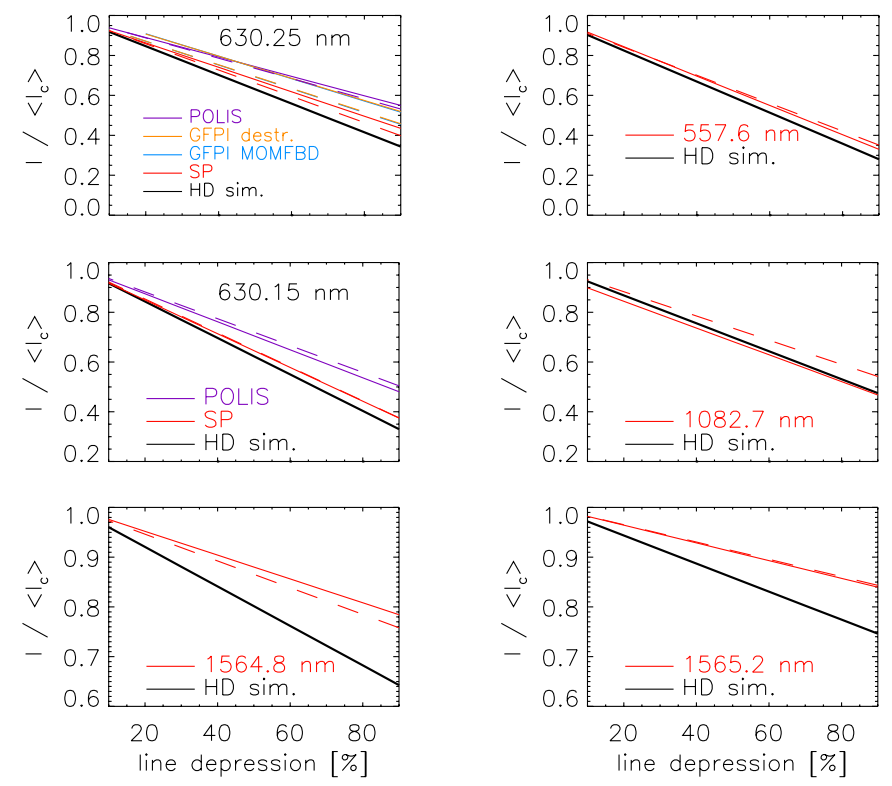

Fig. A.2. Average intensity at different line depression levels in the observations (solid lines), the HD-SPAT-SPEC (dashed lines) and the HDFR (solid black line).
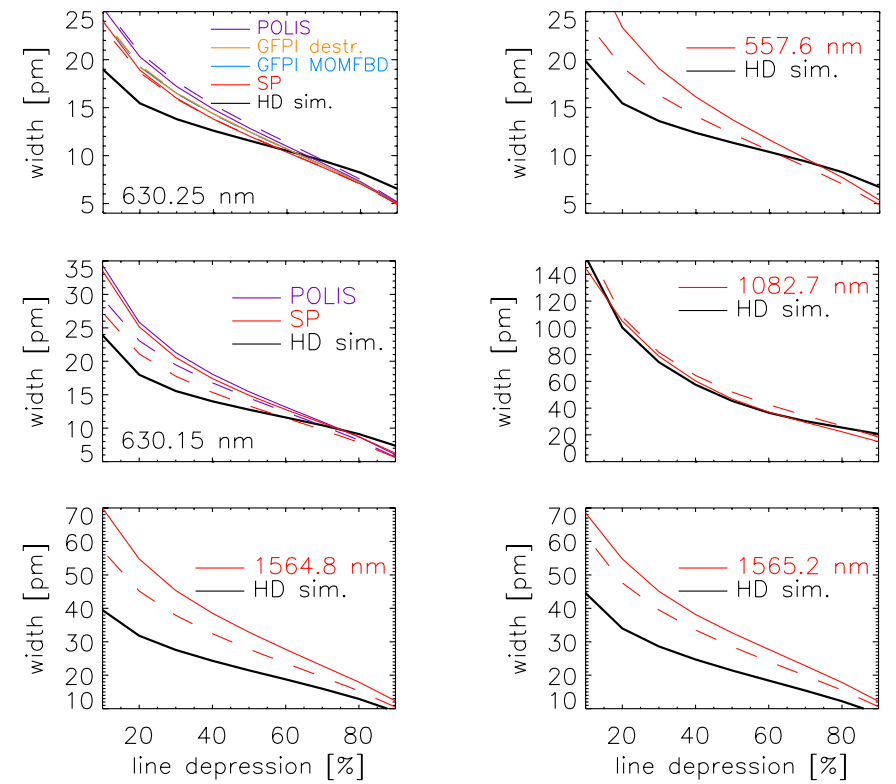

Fig. A.3. Average line width at different line depression levels in the observations (solid lines), the HD-SPAT-SPEC (dashed lines) and the HD-FR (solid black line).

\section{A.2. Rms fluctuations}

The rms fluctuations in the line properties at different line depression levels for all but the $630.25 \mathrm{~nm}$ and $557.6 \mathrm{~nm}$ lines are shown in Fig. A.4. The curves are all to some extent similar to the corresponding ones of the $630.25 \mathrm{~nm}$ line that are described in Sect. 5.3. The most noteworthy feature is that the fluctuations in the HD-SPAT-SPEC never significantly exceed the observed fluctuations in any of the parameters, but rather tend to show slightly lower values. Some parameters show a quite close match between observations and the degraded HD simulation, e.g. the rms velocities for all lines or the intensity rms for the near-IR lines. 

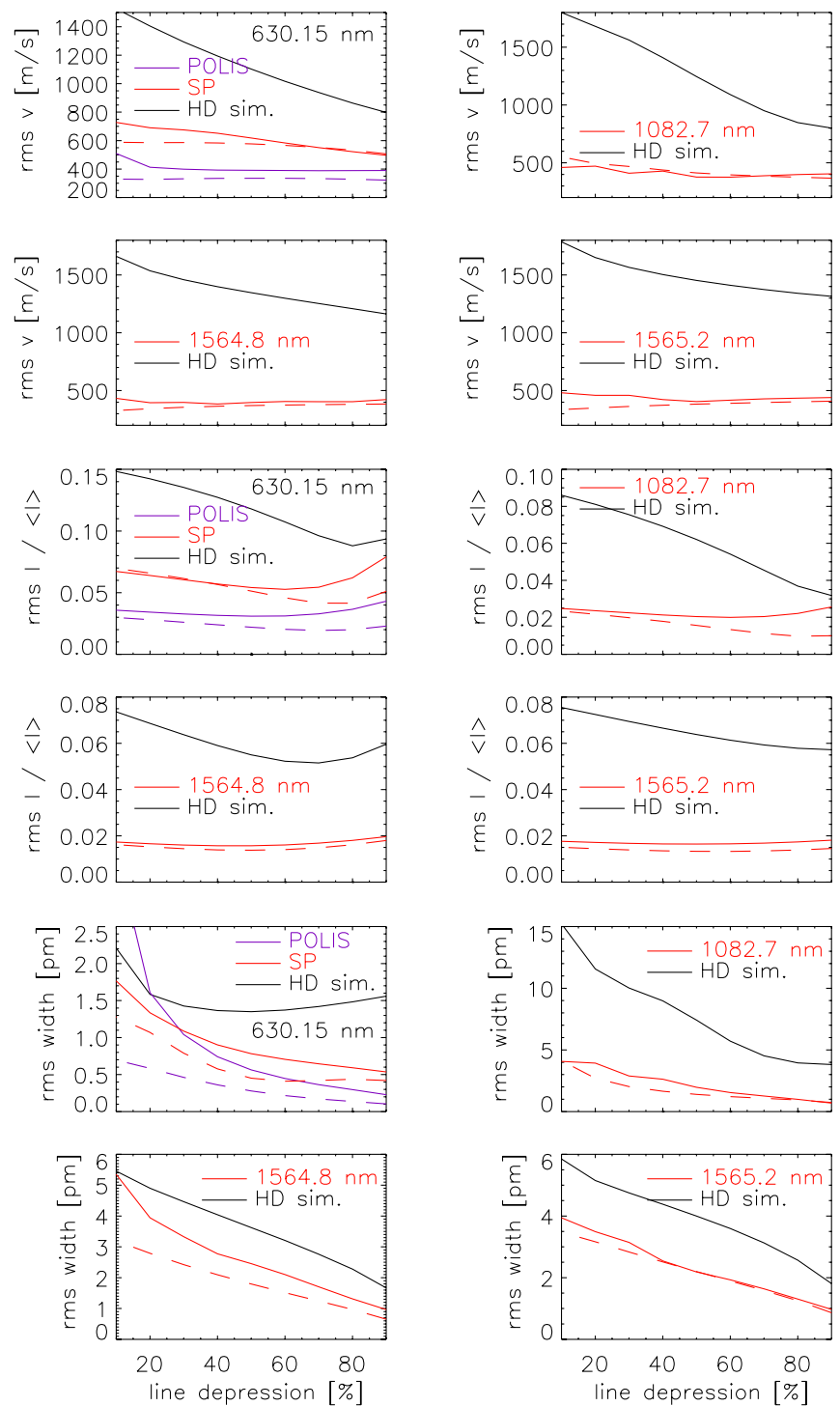

Fig. A.4. Rms fluctuations in the line properties at different line depression levels for all lines but $630.25 \mathrm{~nm}$ and $557.6 \mathrm{~nm}$ in the observations (solid lines), the HD-SPAT-SPEC (dashed lines) and the HD-FR (solid black line). Top two rows: velocity. Middle two rows: intensity. Bottom two rows: line width.

\section{Appendix B: Line parameters of $557.6 \mathrm{~nm}$}

Figure B.1 shows histograms of all line parameters of the Fe I line at $557.6 \mathrm{~nm}$ for completeness. The $557 \mathrm{~nm}$ data were intended to be used for an abundance determination and were therefore recorded even under bad seeing conditions. This quite limits their usefulness for deriving spatial variations of line parameters because all observed spectra already represent some large-scale spatial average. The line is the only one in the sample with a Landé factor of zero, i.e. not sensitive to Zeeman broadening. It thus only reacts to the thermodynamic properties of the atmosphere and not directly to the magnetic field. The line parameters (Fig. B.1) show some deviation between the observations and the HD-SPAT-SPEC, especially the FWHM and the equivalent width. This is most likely related more to the settings in the spectral synthesis, i.e. the method for the inclusion of line broadening, than the thermodynamics themselves. The rms fluctuations in the line properties at different line depression levels (Fig. B.2) in observations and the
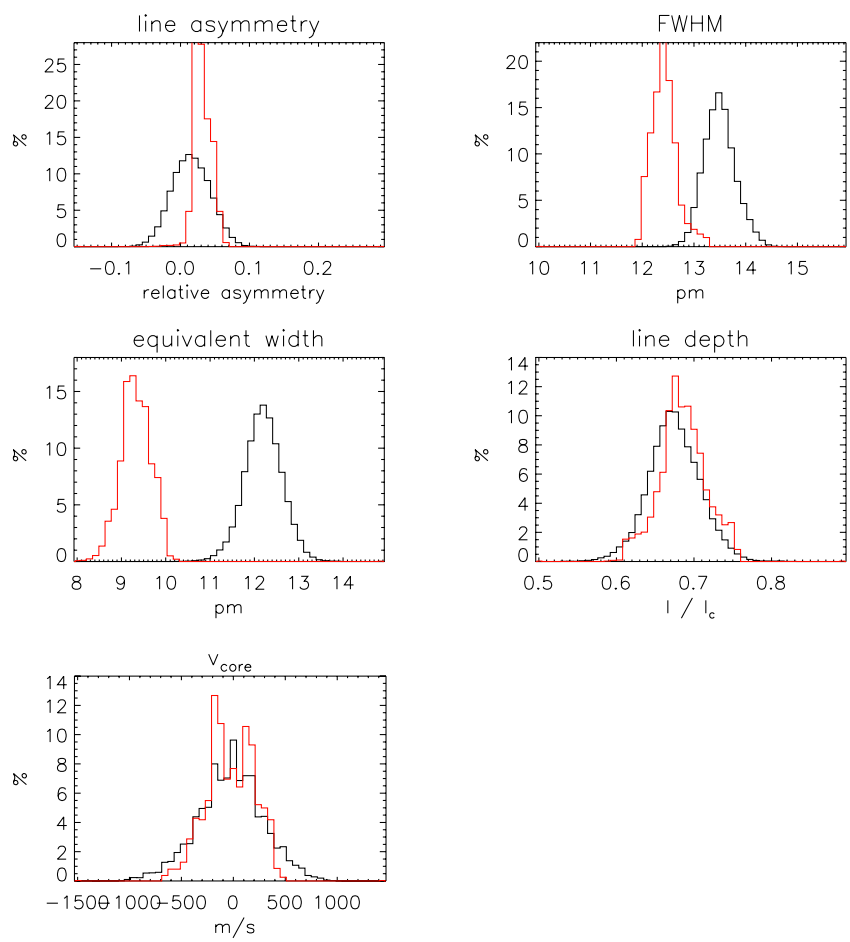

Fig. B.1. Histograms of the line parameters for the $557.6 \mathrm{~nm}$ line. Black lines: observations. Red lines: degraded HD simulation.
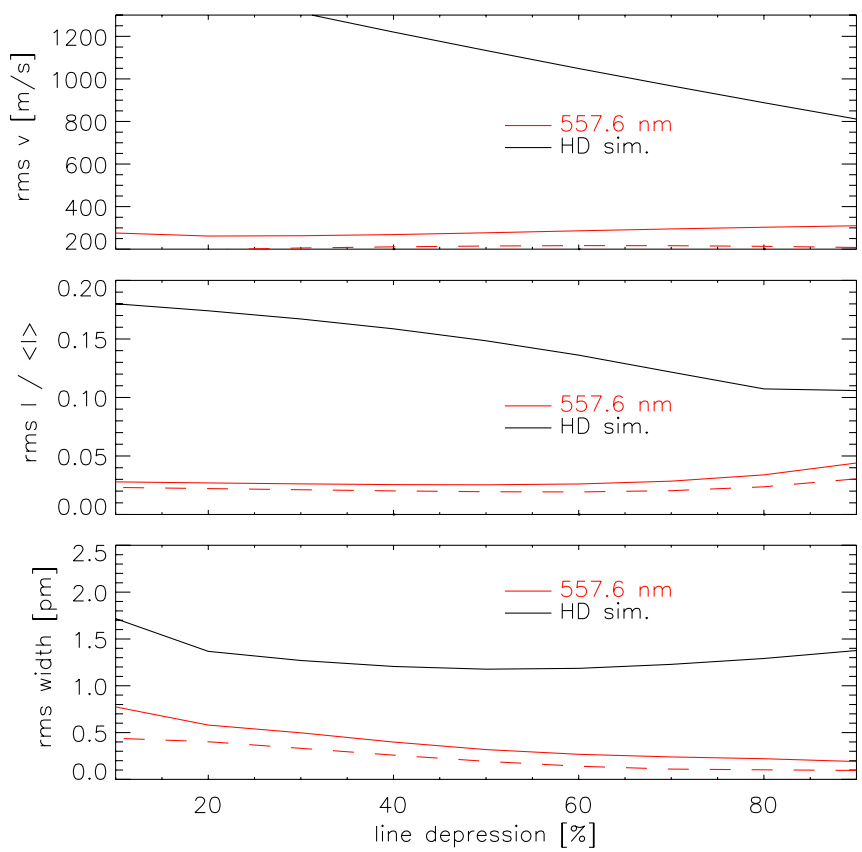

Fig. B.2. Rms fluctuations in the line properties at different line depression levels for the $557.6 \mathrm{~nm}$ line in the observations (solid lines), the HD-SPAT-SPEC (dashed lines) and the HD-FR (solid black line). Top: bisector velocity. Middle: bisector intensity. Bottom: line width.

HD-SPAT-SPEC are more similar than the histograms of line parameters. They show again lower fluctuations in the HD-SPATSPEC than in the observations. The $557.6 \mathrm{~nm}$ line will be most important in the comparison of the thermodynamic characteristics between the field-free, purely HD and the MHD simulations of increasing average magnetic flux. In the present context, this line shows no prominent deviations from the other Zeemansensitive lines in its behaviour. 